\title{
Backscattering enhancement of an electromagnetic wave scattered by two-dimensional rough layers
}

\author{
Antoine Soubret, ${ }^{1,2}$ Gérard Berginc, ${ }^{1}$ and Claude Bourrely ${ }^{2}$ \\ ${ }^{1}$ Thales Optronique, BP 55, 78233 Guyancourt Cedex, France. \\ ${ }^{2}$ Centre de Physique Théorique, CNRS-Luminy Case 907, 13288 Marseille Cedex 9, France.
}

(Dated: December 2000)

\begin{abstract}
The problem of an electromagnetic wave scattering by a slab with two rough boundaries is solved by a small-perturbation method under the Rayleigh hypothesis. In order to obtain a perturbative development, we use a systematic procedure which involves integral equations called the reduced Rayleigh equations. Then we will show for a dielectric slab deposited on a silver film that the backscattering enhancement can be produced by guided waves which interact with the two rough surfaces.
\end{abstract}

\section{INTRODUCTION}

Scattering of electromagnetic waves from multilayer structures is a phenomenon which is of interest in many area of physics such as remote sensing or optical industry, where for example metallic surfaces have a dielectric coating. An extended review of experimental and theoretical wofferen optical multilayers

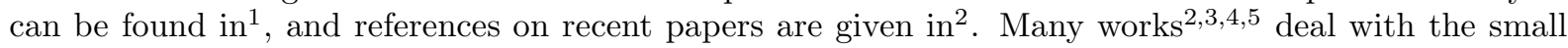
perturbation method to investigate the behaviour of multilayer structure. However, due to calculations complexity, the analytical results are given only to first order in the rms-height of each rough boundary, thus the ipteraction between the rough surfaces can only be taken into account if the rough surfaces are correlated $\mathbf{3}$ in the mean procedure. Our purpose is to show how light can interact with several rough surfaces, to this end, we have choosen the most simple system depicted in Fig. 1, where we have three regions with different permitivities separated by two rough surfaces. The calculations have been made under the hypothesis of the small perturbation method, initially developped by Ricel 6 . Due to the great complexity to derive high orders of the perturbative development, Rice's original method is rather difficult to apply. One way to overcome these difficulties is to use integral equations called the reduced Rayleigh equations, they were first obtained by Celli et a 8 , and later have been generalized in Ref. 9 to take into account upward fields in the slab medium. When combining these equations we obtain an integral equation where only the scattering matrix of the whole structure has to be determined, next we have developed a systematic method to calculate the perturbative development. We have shown by means of numerical simulations, how different mechanisper responsible of the enhanced backscattering occur. This phenomenon which has been predicted 10.11 12.13 and observedes in the case of a random metal surface, manifests itself as a well-defined peak in the retroreflection direction through the angular dependence of the intensity related to the diffuse component of the scattered field. For a metallic surface, the phenomenon is produced by the interference of waves which excite surface plasmon polariton along a certain path and then follow the same path but in the reverse direction. For a dielectric bounded by metallic plate with one rough surface 15 , the enhanced backscattering is produced by a similar mechanism where the surface plasmon polariton is replaced by a slab guided wave. In this case the incident wave excites in a first time a guided mode due to surface roughness, and then the roughness transforms the surface wave into a bulk wave. Furthermore, if the slab supports several guided modes, recent investigations 15 have shown the presence of additionnal peaks, called satellite peaks, in the angular distribution of the incoherent intensity. When the slab has two rough boundaries, the enhancement of backscattering can be produced by two kinds of interaction. The classic one, where the wave is scattered two times by the same boundary, and a new one (see Fig. 11) where the wave is transmitted at a point $A$ of the surface without being diffused, then the wave is scattered by the second rough surface at a point $B$ and by the first one in $C$. If the wave follows the same path in the reverse direction and excites the same guided mode, then phase difference between the two path is $\Delta \phi=\boldsymbol{r}_{B C} \cdot\left(\boldsymbol{k}+\boldsymbol{k}_{i}\right)$, where $\boldsymbol{r}_{B C}$ is the distance between $B$ and $C$. Thus, in the anti-specular direction $\left(\boldsymbol{k}=-\boldsymbol{k}_{i}\right)$, this phase difference is independent of the random position of $B$ and $C$ which produce the backscattering peak.

The outline of the paper is as follows. In Section III we present the system under study and a description of the rough surfaces statistics. In Section III we define the plane wave representation of the electric field in the polarization basis and also the scattering matrix. Section IV is devoted to the calculation of 
the incoherent cross-section as a function of the perturbative development. In Section $\square$ we derive an integral equation from the reduced Rayleigh equations, which provides a systematic way to obtain the perturbative development. The resulting expressions are numerically computed in the case of a dielectric slab deposited on a rough silver surface in Section VI. The calculated incoherent intensity will show a narrow enhanced backscattering. Conclusions drawn from our results are presented and discussed in Section VII. In an Appendix, we collect matrices used in the calculations.

\section{THE RANDOM SURFACE}

The system which is considered in this paper is depicted in Fig. 2.

The three regions are characterized by an isotropic, homogeous dielectric constant $\epsilon_{0}, \epsilon_{1}$ and $\epsilon_{2}$ respectively. The two boundaries are located at $z=h_{1}(\boldsymbol{x})$ and $z=-H+h_{2}(\boldsymbol{x}), \boldsymbol{x}=(x, y)$, and these three media are separated by rough surfaces described statistically. In fact, we assume that $h_{1}(\boldsymbol{x})$ and $h_{2}(\boldsymbol{x})$ are stationary, isotropic uncorrelated Gaussian random processes defined by their moments :

$$
\begin{aligned}
<h_{i}(\boldsymbol{x}) & >=0, \\
<h_{i}(\boldsymbol{x}) h_{i}\left(\boldsymbol{x}^{\prime}\right)> & =W_{i}\left(\boldsymbol{x}-\boldsymbol{x}^{\prime}\right), \\
<h_{1}(\boldsymbol{x}) h_{2}\left(\boldsymbol{x}^{\prime}\right)> & =0
\end{aligned}
$$

where $i=1,2$, and the angle brackets denote an average over the ensemble of realizations of the function $h_{1}(\boldsymbol{x})$ and $h_{2}(\boldsymbol{x})$. In this work we will use a Gaussian form for the surface-height correlation function $W_{1}(\boldsymbol{x})$ and $W_{2}(\boldsymbol{x})$ :

$$
W_{i}(\boldsymbol{x})=\sigma_{i}^{2} \exp \left(-\boldsymbol{x}^{2} / l_{i}^{2}\right)
$$

where $\sigma_{i}$ is the rms height of the surface $h_{i}(\boldsymbol{x})$, and $l_{i}$ is the transverse correlation length. In momentum space we have:

$$
\begin{aligned}
<h_{i}(\boldsymbol{p})> & =0, \\
<h_{i}(\boldsymbol{p}) h_{i}\left(\boldsymbol{p}^{\prime}\right)> & =(2 \pi)^{2} \delta\left(\boldsymbol{p}+\boldsymbol{p}^{\prime}\right) W_{i}(\boldsymbol{p}), \\
<h_{1}(\boldsymbol{p}) h_{2}\left(\boldsymbol{p}^{\prime}\right)> & =0 .
\end{aligned}
$$

with

$$
\begin{aligned}
W_{i}(\boldsymbol{p}) & \equiv \int \mathrm{d}^{2} \boldsymbol{x} W_{i}(\boldsymbol{x}) \exp (-i \boldsymbol{p} \cdot \boldsymbol{x}) \\
& =\pi \sigma_{i}^{2} l_{i}^{2} \exp \left(-\boldsymbol{p}^{2} l_{i}^{2} / 4\right)
\end{aligned}
$$

\section{THE SCATTERING MATRIX}

We suppose that the slab is illuminated from the media 0 by an electromagnetic wave of pulsation $\omega$. In the following we will omit the time dependence $\exp (-\mathrm{i} \omega t)$. The field $\boldsymbol{E}^{0}$ in the media 0 can be written as a superposition of an incident and scattered fields :

$$
\boldsymbol{E}^{0}(\boldsymbol{x}, z)=\boldsymbol{E}^{i}\left(\boldsymbol{p}_{0}\right) \exp \left(\mathrm{i} \boldsymbol{p}_{0} \cdot \boldsymbol{x}-\mathrm{i} \alpha_{0}\left(\boldsymbol{p}_{0}\right) z\right)+\int \frac{\mathrm{d}^{2} \boldsymbol{p}}{(2 \pi)^{2}} \boldsymbol{E}^{s}(\boldsymbol{p}) \exp \left(\mathrm{i} \boldsymbol{p} \cdot \boldsymbol{x}+\mathrm{i} \alpha_{0}(\boldsymbol{p}) z\right) .
$$

where

$$
\begin{aligned}
\alpha_{0}(\boldsymbol{p}) & \equiv\left(\epsilon_{0} K_{0}^{2}-\boldsymbol{p}^{2}\right)^{\frac{1}{2}} \\
\boldsymbol{E}^{i}\left(\boldsymbol{p}_{0}\right) & =E_{V}^{i}\left(\boldsymbol{p}_{0}\right) \hat{\boldsymbol{e}}_{V}^{0-}\left(\boldsymbol{p}_{0}\right)+E_{H}^{i}\left(\boldsymbol{p}_{0}\right) \hat{\boldsymbol{e}}_{H}\left(\boldsymbol{p}_{0}\right) \\
\boldsymbol{E}^{s}(\boldsymbol{p}) & =E_{V}^{s}(\boldsymbol{p}) \hat{\boldsymbol{e}}_{V}^{0+}(\boldsymbol{p})+E_{H}^{s}(\boldsymbol{p}) \hat{\boldsymbol{e}}_{H}(\boldsymbol{p})
\end{aligned}
$$


The subscript $H$ refers to the horizontal polarization $(T E)$ and $V$ to the vertical polarization $(T M)$, and are defined by the two vectors:

$$
\begin{aligned}
\hat{\boldsymbol{e}}_{H}(\boldsymbol{p}) & =\hat{\boldsymbol{e}}_{z} \times \hat{\boldsymbol{p}} \\
\hat{\boldsymbol{e}}_{V}^{0 \pm}(\boldsymbol{p}) & = \pm \frac{\alpha_{0}(\boldsymbol{p})}{\sqrt{\epsilon_{0}} K_{0}} \hat{\boldsymbol{p}}-\frac{\|\boldsymbol{p}\|}{\sqrt{\epsilon_{0}} K_{0}} \hat{\boldsymbol{e}}_{z}
\end{aligned}
$$

where the minus sign refers to incident wave and the plus sign to the scattered wave. It has to be noticed that the vector $\boldsymbol{E}^{s}(\boldsymbol{p})$ and $\boldsymbol{E}^{i}\left(\boldsymbol{p}_{0}\right)$ are expressed in different basis due the fact that $\hat{\boldsymbol{e}}_{V}^{0 \pm}(\boldsymbol{p})$ and $\hat{\boldsymbol{e}}_{V}^{1 \pm}(\boldsymbol{p})$ depend on $\boldsymbol{p}$. In medium 1 , we have a similar expression namely:

$$
\boldsymbol{E}^{1}(\boldsymbol{r})=\int \frac{\mathrm{d}^{2} \boldsymbol{p}}{(2 \pi)^{2}} \boldsymbol{E}^{1-}(\boldsymbol{p}) \exp \left(\mathrm{i} \boldsymbol{p} \cdot \boldsymbol{x}-\mathrm{i} \alpha_{1}(\boldsymbol{p}) z\right)+\int \frac{\mathrm{d}^{2} \boldsymbol{p}}{(2 \pi)^{2}} \boldsymbol{E}^{1+}(\boldsymbol{p}) \exp \left(\mathrm{i} \boldsymbol{p} \cdot \boldsymbol{x}+\mathrm{i} \alpha_{1}(\boldsymbol{p}) z\right)
$$

where

$$
\alpha_{1}(\boldsymbol{p}) \equiv\left(\epsilon_{1} K_{0}^{2}-\boldsymbol{p}^{2}\right)^{\frac{1}{2}}
$$

The field $\boldsymbol{E}^{1-}$ is decomposed in the basis $\left(\hat{\boldsymbol{e}}_{V}^{-}(\boldsymbol{p}), \hat{\boldsymbol{e}}_{H}(\boldsymbol{p})\right)$, and $\boldsymbol{E}^{1+}$ in the basis $\left(\hat{\boldsymbol{e}}_{V}^{+}(\boldsymbol{p}), \hat{\boldsymbol{e}}_{H}(\boldsymbol{p})\right)$ with

$$
\begin{aligned}
\hat{\boldsymbol{e}}_{H}(\boldsymbol{p}) & =\hat{\boldsymbol{e}}_{z} \times \hat{\boldsymbol{p}} \\
\hat{\boldsymbol{e}}_{V}^{1 \pm}(\boldsymbol{p}) & = \pm \frac{\alpha_{1}(\boldsymbol{p})}{\sqrt{\epsilon_{1}} K_{0}} \hat{\boldsymbol{p}}-\frac{\|\boldsymbol{p}\|}{\sqrt{\epsilon_{1}} K_{0}} \hat{\boldsymbol{e}}_{z} .
\end{aligned}
$$

We now introduce the definition the scattering matrix :

$$
\boldsymbol{E}^{s}(\boldsymbol{p}) \equiv \overline{\boldsymbol{R}}\left(\boldsymbol{p} \mid \boldsymbol{p}_{0}\right) \cdot \boldsymbol{E}^{i}\left(\boldsymbol{p}_{0}\right)
$$

where $\overline{\boldsymbol{R}}\left(\boldsymbol{p} \mid \boldsymbol{p}_{0}\right)$ is a two dimensional matrix which can be written in the following form:

$$
\overline{\boldsymbol{R}}\left(\boldsymbol{p} \mid \boldsymbol{p}_{0}\right)=\left(\begin{array}{ll}
R_{V V}\left(\boldsymbol{p} \mid \boldsymbol{p}_{0}\right) & R_{V H}\left(\boldsymbol{p} \mid \boldsymbol{p}_{0}\right) \\
R_{H V}\left(\boldsymbol{p} \mid \boldsymbol{p}_{0}\right) & R_{H H}\left(\boldsymbol{p} \mid \boldsymbol{p}_{0}\right)
\end{array}\right)
$$

\section{DIFFUSE CROSS-SECTION}

In a previous work 9 we have defined a new product $\odot$ between two dimensional matrices in the form :

$$
\begin{aligned}
& \overline{\boldsymbol{f}} \odot \overline{\boldsymbol{g}}=\left(\begin{array}{ll}
f_{V V} & f_{V H} \\
f_{H V} & f_{H H}
\end{array}\right) \odot\left(\begin{array}{ll}
g_{V V} & g_{V H} \\
g_{H V} & g_{H H}
\end{array}\right) \\
& \equiv\left(\begin{array}{cccc}
f_{V V} g_{V V}^{*} & f_{V H} g_{V H}^{*} & \operatorname{Re}\left(f_{V V} g_{V H}^{*}\right) & -\operatorname{Im}\left(f_{V V} g_{V H}^{*}\right) \\
f_{H V} g_{H V}^{*} & f_{H H} g_{H H}^{*} & \operatorname{Re}\left(f_{H V} g_{H H}^{*}\right) & -\operatorname{Im}\left(f_{H V} g_{V H}^{*}\right) \\
2 R e\left(f_{V V} g_{H V}^{*}\right) & 2 R e\left(f_{V H} g_{H H}^{*}\right) & \operatorname{Re}\left(f_{V V} g_{V V}^{*}+f_{H V} g_{V H}^{*}\right) & -\operatorname{Im}\left(f_{V V} g_{H H}^{*}-f_{V H} g_{H V}^{*}\right) \\
2 \operatorname{Im}\left(f_{V V} g_{H V}^{*}\right) & 2 \operatorname{Im}\left(f_{V H} g_{H H}^{*}\right) & \operatorname{Im}\left(f_{V V} g_{V V}^{*}+f_{H V} g_{V H}^{*}\right) & \operatorname{Re}\left(f_{V V} g_{H H}^{*}-f_{V H} g_{H V}^{*}\right)
\end{array}\right) .
\end{aligned}
$$

which allows to write the incoherent Muller matrix 16 in a condensed expression:

$$
\overline{\boldsymbol{M}}^{\text {incoh }}\left(\boldsymbol{p} \mid \boldsymbol{p}_{0}\right)=\frac{K_{0}^{2} \cos ^{2} \theta}{(2 \pi)^{2}}\left[<\overline{\boldsymbol{R}}\left(\boldsymbol{p} \mid \boldsymbol{p}_{0}\right) \odot \overline{\boldsymbol{R}}\left(\boldsymbol{p} \mid \boldsymbol{p}_{0}\right)>-<\overline{\boldsymbol{R}}\left(\boldsymbol{p} \mid \boldsymbol{p}_{0}\right)>\odot<\overline{\boldsymbol{R}}\left(\boldsymbol{p} \mid \boldsymbol{p}_{0}\right)>\right]
$$

Furthermore, we define the generalization of the classical bistatic coefficient $₫$ also called mean differential coefficient 18 by :

$$
\overline{\boldsymbol{\gamma}}^{\text {incoh }}\left(\boldsymbol{p} \mid \boldsymbol{p}_{0}\right) \equiv \frac{1}{A \cos \theta_{0}} \overline{\boldsymbol{M}}^{\text {incoh }}\left(\boldsymbol{p} \mid \boldsymbol{p}_{0}\right)
$$

where $A$ is the area of the illuminated surface and $\theta_{0}$ the incident angle (see Fig. 3). In this paper we are interested by the perturbative development of the scattered fields as a function of the surface elevations 
$h_{1}$ and $h_{2}$. In a perturbative expansion of the scattering matrix, the terms which contain an expression like $h_{1}^{n} h_{2}^{m}$ will be denoted $\overline{\boldsymbol{R}}^{(n m)}$, so that the perturbative development of $\overline{\boldsymbol{R}}$ becomes:

$$
\overline{\boldsymbol{R}}=\overline{\boldsymbol{R}}^{(00)}+\overline{\boldsymbol{R}}^{(10)}+\overline{\boldsymbol{R}}^{(01)}+\overline{\boldsymbol{R}}^{(11)}+\overline{\boldsymbol{R}}^{(20)}+\overline{\boldsymbol{R}}^{(21)}+\overline{\boldsymbol{R}}^{(12)}+\overline{\boldsymbol{R}}^{(22)}+\overline{\boldsymbol{R}}^{(30)}+\overline{\boldsymbol{R}}^{(03)}+\ldots
$$

With this decomposition and using Eqs. (1.:3) with the fact that $h_{1}$ and $h_{2}$ are Gaussian random processes i.e.

$$
\begin{aligned}
& <h_{1}^{2 p+1}(\boldsymbol{x})>=0, \quad p \text { a positive integer } \\
& <h_{2}^{2 p+1}(\boldsymbol{x})>=0
\end{aligned}
$$

the incoherent bistatic matrix will be given by the contribution of three terms:

$$
\overline{\boldsymbol{\gamma}}^{\text {incoh }}\left(\boldsymbol{p} \mid \boldsymbol{p}_{0}\right)=\overline{\boldsymbol{\gamma}}_{u}^{\text {incoh }}\left(\boldsymbol{p} \mid \boldsymbol{p}_{0}\right)+\overline{\boldsymbol{\gamma}}_{d}^{\text {incoh }}\left(\boldsymbol{p} \mid \boldsymbol{p}_{0}\right)+\overline{\boldsymbol{\gamma}}_{u d}^{\text {incoh }}\left(\boldsymbol{p} \mid \boldsymbol{p}_{0}\right)
$$

where

$$
\overline{\boldsymbol{\gamma}}_{u}^{\text {incoh }}\left(\boldsymbol{p} \mid \boldsymbol{p}_{0}\right)=\frac{K_{0}^{2} \cos ^{2} \theta}{A(2 \pi)^{2} \cos \theta_{0}}\left[<\overline{\boldsymbol{R}}^{(10)} \odot \overline{\boldsymbol{R}}^{(10)}>+<\overline{\boldsymbol{R}}^{(20)} \odot \overline{\boldsymbol{R}}^{(20)}>+<\overline{\boldsymbol{R}}^{(30)} \odot \overline{\boldsymbol{R}}^{(10)}>\right],
$$

corresponds to the incoherent bistatic matrix for the slab where only the upper surface has a roughness $\left(h_{2}(\boldsymbol{x})=0\right)$, and its expansion is made up to order four in the rms-height elevation $\sigma_{1}$. Similarly,

$$
\overline{\boldsymbol{\gamma}}_{d}^{\text {incoh }}\left(\boldsymbol{p} \mid \boldsymbol{p}_{0}\right)=\frac{K_{0}^{2} \cos ^{2} \theta}{A(2 \pi)^{2} \cos \theta_{0}}\left[<\overline{\boldsymbol{R}}^{(01)} \odot \overline{\boldsymbol{R}}^{(01)}>+<\overline{\boldsymbol{R}}^{(02)} \odot \overline{\boldsymbol{R}}^{(02)}>+<\overline{\boldsymbol{R}}^{(03)} \odot \overline{\boldsymbol{R}}^{(01)}>\right],
$$

is associated to a system where only the bottom surface is rough $\left(h_{1}(\boldsymbol{x})=0\right)$, where also the perturbative development is made up to order four in $\sigma_{2}$. The last term $\bar{\gamma}_{u d}^{\text {incoh }}$ contains terms which describe the scattering process between the two rough surfaces where only the leading terms are retained:

$$
\begin{aligned}
\overline{\boldsymbol{\gamma}}_{u d}^{\text {incoh }}\left(\boldsymbol{p} \mid \boldsymbol{p}_{0}\right)=\frac{K_{0}^{2} \cos ^{2} \theta}{A(2 \pi)^{2} \cos \theta_{0}}[ & <\overline{\boldsymbol{R}}^{(10)} \odot \overline{\boldsymbol{R}}^{(12)}>+<\overline{\boldsymbol{R}}^{(12)} \odot \overline{\boldsymbol{R}}^{(10)}> \\
& +<\overline{\boldsymbol{R}}^{(01)} \odot \overline{\boldsymbol{R}}^{(21)}>+<\overline{\boldsymbol{R}}^{(21)} \odot \overline{\boldsymbol{R}}^{(01)}> \\
& \left.+<\overline{\boldsymbol{R}}^{(11)} \odot \overline{\boldsymbol{R}}^{(11)}>+\ldots\right]
\end{aligned}
$$

More precisely all terms up of order $\sigma_{1}^{2} \sigma_{2}^{2}$ are included. If the value of $\sigma_{1}$ and $\sigma_{2}$ are of the same order of magnitude, these terms will be comparable to the order four in the expressions (28,29). Thus we can suppose that the following terms in the expansion (30), which are of order $\sigma_{1}^{4} \sigma_{2}^{2}, \sigma_{1}^{2} \sigma_{2}^{4}, \sigma_{1}^{4} \sigma_{2}^{4}$ will be negligible compared to those retained in Eq. (30). However, due to the complexity of the perturbative development, these terms of the sixth order have not been calculated. In the following section, we will show how the perturbative development can be put in the following form:

$$
\begin{gathered}
\overline{\boldsymbol{R}}^{(10)}\left(\boldsymbol{p} \mid \boldsymbol{p}_{0}\right)=\alpha_{0}\left(\boldsymbol{p}_{0}\right) \overline{\boldsymbol{X}}^{(10)}\left(\boldsymbol{p} \mid \boldsymbol{p}_{0}\right) h_{1}\left(\boldsymbol{p}-\boldsymbol{p}_{0}\right) \\
\overline{\boldsymbol{R}}^{(01)}\left(\boldsymbol{p} \mid \boldsymbol{p}_{0}\right)=\alpha_{0}\left(\boldsymbol{p}_{0}\right) \overline{\boldsymbol{X}}^{(01)}\left(\boldsymbol{p} \mid \boldsymbol{p}_{0}\right) h_{2}\left(\boldsymbol{p}-\boldsymbol{p}_{0}\right) \\
\overline{\boldsymbol{R}}^{(11)}\left(\boldsymbol{p} \mid \boldsymbol{p}_{0}\right)=\alpha_{0}\left(\boldsymbol{p}_{0}\right) \int \frac{\mathrm{d}^{2} \boldsymbol{p}_{1}}{(2 \pi)^{2}}\left[\overline{\boldsymbol{X}}^{(11) 12}\left(\boldsymbol{p}\left|\boldsymbol{p}_{1}\right| \boldsymbol{p}_{0}\right) h_{1}\left(\boldsymbol{p}-\boldsymbol{p}_{1}\right) h_{2}\left(\boldsymbol{p}-\boldsymbol{p}_{0}\right)\right. \\
\left.+\overline{\boldsymbol{X}}^{(11) 21}\left(\boldsymbol{p}\left|\boldsymbol{p}_{1}\right| \boldsymbol{p}_{0}\right) h_{2}\left(\boldsymbol{p}-\boldsymbol{p}_{1}\right) h_{1}\left(\boldsymbol{p}-\boldsymbol{p}_{0}\right)\right] \\
\overline{\boldsymbol{R}}^{(20)}\left(\boldsymbol{p} \mid \boldsymbol{p}_{0}\right)=\alpha_{0}\left(\boldsymbol{p}_{0}\right) \int \frac{\mathrm{d}^{2} \boldsymbol{p}_{1}}{(2 \pi)^{2}} \overline{\boldsymbol{X}}^{(20)}\left(\boldsymbol{p}\left|\boldsymbol{p}_{1}\right| \boldsymbol{p}_{0}\right) h_{1}\left(\boldsymbol{p}-\boldsymbol{p}_{1}\right) h_{1}\left(\boldsymbol{p}_{1}-\boldsymbol{p}_{0}\right) \\
\overline{\boldsymbol{R}}^{(02)}\left(\boldsymbol{p} \mid \boldsymbol{p}_{0}\right)=\alpha_{0}\left(\boldsymbol{p}_{0}\right) \int \frac{\mathrm{d}^{2} \boldsymbol{p}_{1}}{(2 \pi)^{2}} \overline{\boldsymbol{X}}^{(02)}\left(\boldsymbol{p}\left|\boldsymbol{p}_{1}\right| \boldsymbol{p}_{0}\right) h_{2}\left(\boldsymbol{p}-\boldsymbol{p}_{1}\right) h_{2}\left(\boldsymbol{p}_{1}-\boldsymbol{p}_{0}\right),
\end{gathered}
$$




$$
\begin{aligned}
\overline{\boldsymbol{R}}^{(21)}\left(\boldsymbol{p} \mid \boldsymbol{p}_{0}\right)=\alpha_{0}\left(\boldsymbol{p}_{0}\right) \iint \frac{\mathrm{d}^{2} \boldsymbol{p}_{1}}{(2 \pi)^{2}} \frac{\mathrm{d}^{2} \boldsymbol{p}_{2}}{(2 \pi)^{2}} & {\left[\overline{\boldsymbol{X}}^{(21) 112}\left(\boldsymbol{p}\left|\boldsymbol{p}_{1}\right| \boldsymbol{p}_{2} \mid \boldsymbol{p}_{0}\right) h_{1}\left(\boldsymbol{p}-\boldsymbol{p}_{1}\right) h_{1}\left(\boldsymbol{p}_{1}-\boldsymbol{p}_{2}\right) h_{2}\left(\boldsymbol{p}_{2}-\boldsymbol{p}_{0}\right)\right.} \\
& +\overline{\boldsymbol{X}}^{(21) 121}\left(\boldsymbol{p}\left|\boldsymbol{p}_{1}\right| \boldsymbol{p}_{2} \mid \boldsymbol{p}_{0}\right) h_{1}\left(\boldsymbol{p}-\boldsymbol{p}_{1}\right) h_{2}\left(\boldsymbol{p}_{1}-\boldsymbol{p}_{2}\right) h_{1}\left(\boldsymbol{p}_{2}-\boldsymbol{p}_{0}\right) \\
& \left.+\overline{\boldsymbol{X}}^{(21) 211}\left(\boldsymbol{p}\left|\boldsymbol{p}_{1}\right| \boldsymbol{p}_{2} \mid \boldsymbol{p}_{0}\right) h_{2}\left(\boldsymbol{p}-\boldsymbol{p}_{1}\right) h_{1}\left(\boldsymbol{p}_{1}-\boldsymbol{p}_{2}\right) h_{1}\left(\boldsymbol{p}_{2}-\boldsymbol{p}_{0}\right)\right],
\end{aligned}
$$

$$
\begin{aligned}
\overline{\boldsymbol{R}}^{(12)}\left(\boldsymbol{p} \mid \boldsymbol{p}_{0}\right)=\alpha_{0}\left(\boldsymbol{p}_{0}\right) \iint \frac{\mathrm{d}^{2} \boldsymbol{p}_{1}}{(2 \pi)^{2}} \frac{\mathrm{d}^{2} \boldsymbol{p}_{2}}{(2 \pi)^{2}} & {\left[\overline{\boldsymbol{X}}^{(12) 221}\left(\boldsymbol{p}\left|\boldsymbol{p}_{1}\right| \boldsymbol{p}_{2} \mid \boldsymbol{p}_{0}\right) h_{2}\left(\boldsymbol{p}-\boldsymbol{p}_{1}\right) h_{2}\left(\boldsymbol{p}_{1}-\boldsymbol{p}_{2}\right) h_{1}\left(\boldsymbol{p}_{2}-\boldsymbol{p}_{0}\right)\right.} \\
& +\overline{\boldsymbol{X}}^{(12) 212}\left(\boldsymbol{p}\left|\boldsymbol{p}_{1}\right| \boldsymbol{p}_{2} \mid \boldsymbol{p}_{0}\right) h_{2}\left(\boldsymbol{p}-\boldsymbol{p}_{1}\right) h_{1}\left(\boldsymbol{p}_{1}-\boldsymbol{p}_{2}\right) h_{2}\left(\boldsymbol{p}_{2}-\boldsymbol{p}_{0}\right) \\
& \left.+\overline{\boldsymbol{X}}^{(12) 122}\left(\boldsymbol{p}\left|\boldsymbol{p}_{1}\right| \boldsymbol{p}_{2} \mid \boldsymbol{p}_{0}\right) h_{1}\left(\boldsymbol{p}-\boldsymbol{p}_{1}\right) h_{2}\left(\boldsymbol{p}_{1}-\boldsymbol{p}_{2}\right) h_{2}\left(\boldsymbol{p}_{2}-\boldsymbol{p}_{0}\right)\right],
\end{aligned}
$$

$$
\begin{aligned}
& \overline{\boldsymbol{R}}^{(30)}\left(\boldsymbol{p} \mid \boldsymbol{p}_{0}\right)=\alpha_{0}\left(\boldsymbol{p}_{0}\right) \iint \frac{\mathrm{d}^{2} \boldsymbol{p}_{1}}{(2 \pi)^{2}} \frac{\mathrm{d}^{2} \boldsymbol{p}_{2}}{(2 \pi)^{2}} \overline{\boldsymbol{X}}^{(30)}\left(\boldsymbol{p}\left|\boldsymbol{p}_{1}\right| \boldsymbol{p}_{2} \mid \boldsymbol{p}_{0}\right) h_{1}\left(\boldsymbol{p}-\boldsymbol{p}_{1}\right) h_{1}\left(\boldsymbol{p}_{1}-\boldsymbol{p}_{2}\right) h_{1}\left(\boldsymbol{p}_{2}-\boldsymbol{p}_{0}\right) \\
& \overline{\boldsymbol{R}}^{(03)}\left(\boldsymbol{p} \mid \boldsymbol{p}_{0}\right)=\alpha_{0}\left(\boldsymbol{p}_{0}\right) \iint \frac{\mathrm{d}^{2} \boldsymbol{p}_{1}}{(2 \pi)^{2}} \frac{\mathrm{d}^{2} \boldsymbol{p}_{2}}{(2 \pi)^{2}} \overline{\boldsymbol{X}}^{(03)}\left(\boldsymbol{p}\left|\boldsymbol{p}_{1}\right| \boldsymbol{p}_{2} \mid \boldsymbol{p}_{0}\right) h_{2}\left(\boldsymbol{p}-\boldsymbol{p}_{1}\right) h_{2}\left(\boldsymbol{p}_{1}-\boldsymbol{p}_{2}\right) h_{2}\left(\boldsymbol{p}_{2}-\boldsymbol{p}_{0}\right) .
\end{aligned}
$$

In these expressions we have added superscripts in some terms to indicate the order of apparition of the functions $h_{1}$ and $h_{2}$. For example, in $\overline{\boldsymbol{X}}^{(21) 121}$ the superscript 121 indicates that it is the coefficient associated with the product $h_{1}\left(\boldsymbol{p}-\boldsymbol{p}_{1}\right) h_{2}\left(\boldsymbol{p}_{1}-\boldsymbol{p}_{2}\right) h_{1}\left(\boldsymbol{p}_{2}-\boldsymbol{p}_{0}\right)$. When we combine Eq. $(6), \delta(0)=A /(2 \pi)^{2}$ and the previous development, we obtain the following expression for the quantities (28) 30 ):

$$
\begin{aligned}
& \bar{\gamma}_{u}^{\text {incoh }}\left(\boldsymbol{p} \mid \boldsymbol{p}_{0}\right)=\frac{K_{0}^{4} \cos ^{2} \theta \cos \theta_{0}}{(2 \pi)^{2}}\left[\overline{\boldsymbol{I}}^{(10-10)}\left(\boldsymbol{p} \mid \boldsymbol{p}_{0}\right)+\overline{\boldsymbol{I}}^{(20-20)}\left(\boldsymbol{p} \mid \boldsymbol{p}_{0}\right)+\overline{\boldsymbol{I}}^{(30-10)}\left(\boldsymbol{p} \mid \boldsymbol{p}_{0}\right)\right], \\
& \bar{\gamma}_{d}^{\text {incoh }}\left(\boldsymbol{p} \mid \boldsymbol{p}_{0}\right)=\frac{K_{0}^{4} \cos ^{2} \theta \cos \theta_{0}}{(2 \pi)^{2}}\left[\overline{\boldsymbol{I}}^{(01-01)}\left(\boldsymbol{p} \mid \boldsymbol{p}_{0}\right)+\overline{\boldsymbol{I}}^{(02-02)}\left(\boldsymbol{p} \mid \boldsymbol{p}_{0}\right)+\overline{\boldsymbol{I}}^{(03-01)}\left(\boldsymbol{p} \mid \boldsymbol{p}_{0}\right)\right], \\
& \overline{\boldsymbol{\gamma}}_{u d}^{\text {incoh }}\left(\boldsymbol{p} \mid \boldsymbol{p}_{0}\right)=\frac{K_{0}^{4} \cos ^{2} \theta \cos \theta_{0}}{(2 \pi)^{2}}\left[\overline{\boldsymbol{I}}^{(12-10)}\left(\boldsymbol{p} \mid \boldsymbol{p}_{0}\right)+\overline{\boldsymbol{I}}^{(11-11)}\left(\boldsymbol{p} \mid \boldsymbol{p}_{0}\right)+\overline{\boldsymbol{I}}^{(21-01)}\left(\boldsymbol{p} \mid \boldsymbol{p}_{0}\right)\right],
\end{aligned}
$$

where

$$
\begin{aligned}
& \overline{\boldsymbol{I}}^{(10-10)}\left(\boldsymbol{p} \mid \boldsymbol{p}_{0}\right)=W_{1}\left(\boldsymbol{p}-\boldsymbol{p}_{0}\right) \overline{\boldsymbol{X}}^{(10)}\left(\boldsymbol{p} \mid \boldsymbol{p}_{0}\right) \odot \overline{\boldsymbol{X}}^{(10)}\left(\boldsymbol{p} \mid \boldsymbol{p}_{0}\right) \\
& \overline{\boldsymbol{I}}^{(20-20)}\left(\boldsymbol{p} \mid \boldsymbol{p}_{0}\right)=\int \frac{\mathrm{d}^{2} \boldsymbol{p}_{1}}{(2 \pi)^{2}} W_{1}\left(\boldsymbol{p}-\boldsymbol{p}_{1}\right) W_{1}\left(\boldsymbol{p}_{1}-\boldsymbol{p}_{0}\right) \overline{\boldsymbol{X}}^{(20)}\left(\boldsymbol{p}\left|\boldsymbol{p}_{1}\right| \boldsymbol{p}_{0}\right) \\
& \odot\left[\overline{\boldsymbol{X}}^{(20)}\left(\boldsymbol{p}\left|\boldsymbol{p}_{1}\right| \boldsymbol{p}_{0}\right)+\overline{\boldsymbol{X}}^{(20)}\left(\boldsymbol{p}\left|\boldsymbol{p}+\boldsymbol{p}_{0}-\boldsymbol{p}_{1}\right| \boldsymbol{p}_{0}\right)\right] \\
& \overline{\boldsymbol{I}}^{(30-10)}\left(\boldsymbol{p} \mid \boldsymbol{p}_{0}\right)=W_{1}\left(\boldsymbol{p}-\boldsymbol{p}_{0}\right)\left[\overline{\boldsymbol{X}}^{(10)}\left(\boldsymbol{p} \mid \boldsymbol{p}_{0}\right) \odot \overline{\boldsymbol{X}}^{(30)}\left(\boldsymbol{p} \mid \boldsymbol{p}_{0}\right)+\overline{\boldsymbol{X}}^{(30)}\left(\boldsymbol{p} \mid \boldsymbol{p}_{0}\right) \odot \overline{\boldsymbol{X}}^{(10)}\left(\boldsymbol{p} \mid \boldsymbol{p}_{0}\right)\right], \\
& \overline{\boldsymbol{I}}^{(01-01)}\left(\boldsymbol{p} \mid \boldsymbol{p}_{0}\right)=W_{2}\left(\boldsymbol{p}-\boldsymbol{p}_{0}\right) \overline{\boldsymbol{X}}^{(01)}\left(\boldsymbol{p} \mid \boldsymbol{p}_{0}\right) \odot \overline{\boldsymbol{X}}^{(01)}\left(\boldsymbol{p} \mid \boldsymbol{p}_{0}\right) \\
& \overline{\boldsymbol{I}}^{(02-02)}\left(\boldsymbol{p} \mid \boldsymbol{p}_{0}\right)=\int \frac{\mathrm{d}^{2} \boldsymbol{p}_{1}}{(2 \pi)^{2}} W_{2}\left(\boldsymbol{p}-\boldsymbol{p}_{1}\right) W_{2}\left(\boldsymbol{p}_{1}-\boldsymbol{p}_{0}\right) \overline{\boldsymbol{X}}^{(02)}\left(\boldsymbol{p}\left|\boldsymbol{p}_{1}\right| \boldsymbol{p}_{0}\right) \\
& \odot\left[\overline{\boldsymbol{X}}^{(02)}\left(\boldsymbol{p}\left|\boldsymbol{p}_{1}\right| \boldsymbol{p}_{0}\right)+\overline{\boldsymbol{X}}^{(02)}\left(\boldsymbol{p}\left|\boldsymbol{p}+\boldsymbol{p}_{0}-\boldsymbol{p}_{1}\right| \boldsymbol{p}_{0}\right)\right] \\
& \overline{\boldsymbol{I}}^{(03-01)}\left(\boldsymbol{p} \mid \boldsymbol{p}_{0}\right)=W_{2}\left(\boldsymbol{p}-\boldsymbol{p}_{0}\right)\left[\overline{\boldsymbol{X}}^{(01)}\left(\boldsymbol{p} \mid \boldsymbol{p}_{0}\right) \odot \overline{\boldsymbol{X}}^{(03)}\left(\boldsymbol{p} \mid \boldsymbol{p}_{0}\right)+\overline{\boldsymbol{X}}^{(03)}\left(\boldsymbol{p} \mid \boldsymbol{p}_{0}\right) \odot \overline{\boldsymbol{X}}^{(01)}\left(\boldsymbol{p} \mid \boldsymbol{p}_{0}\right)\right],
\end{aligned}
$$




$$
\begin{aligned}
\overline{\boldsymbol{I}}^{(12-10)}\left(\boldsymbol{p} \mid \boldsymbol{p}_{0}\right) & =W_{1}\left(\boldsymbol{p}-\boldsymbol{p}_{0}\right)\left[\overline{\boldsymbol{X}}^{(12)}\left(\boldsymbol{p} \mid \boldsymbol{p}_{0}\right) \odot \overline{\boldsymbol{X}}^{(10)}\left(\boldsymbol{p} \mid \boldsymbol{p}_{0}\right)+\overline{\boldsymbol{X}}^{(10)}\left(\boldsymbol{p} \mid \boldsymbol{p}_{0}\right) \odot \overline{\boldsymbol{X}}^{(12)}\left(\boldsymbol{p} \mid \boldsymbol{p}_{0}\right)\right] \\
\overline{\boldsymbol{I}}^{(21-01)}\left(\boldsymbol{p} \mid \boldsymbol{p}_{0}\right) & =W_{2}\left(\boldsymbol{p}-\boldsymbol{p}_{0}\right)\left[\overline{\boldsymbol{X}}^{(21)}\left(\boldsymbol{p} \mid \boldsymbol{p}_{0}\right) \odot \overline{\boldsymbol{X}}^{(01)}\left(\boldsymbol{p} \mid \boldsymbol{p}_{0}\right)+\overline{\boldsymbol{X}}^{(01)}\left(\boldsymbol{p} \mid \boldsymbol{p}_{0}\right) \odot \overline{\boldsymbol{X}}^{(21)}\left(\boldsymbol{p} \mid \boldsymbol{p}_{0}\right)\right] \\
\overline{\boldsymbol{I}}^{(11-11)}\left(\boldsymbol{p} \mid \boldsymbol{p}_{0}\right) & =\int \frac{\mathrm{d}^{2} \boldsymbol{p}_{1}}{(2 \pi)^{2}}\left[W_{1}\left(\boldsymbol{p}-\boldsymbol{p}_{1}\right) W_{2}\left(\boldsymbol{p}_{1}-\boldsymbol{p}_{0}\right) \overline{\boldsymbol{X}}^{(11) 12}\left(\boldsymbol{p}\left|\boldsymbol{p}_{1}\right| \boldsymbol{p}_{0}\right)\right. \\
& \odot\left(\overline{\boldsymbol{X}}^{(11) 12}\left(\boldsymbol{p}\left|\boldsymbol{p}_{1}\right| \boldsymbol{p}_{0}\right)+\overline{\boldsymbol{X}}^{(11) 21}\left(\boldsymbol{p}\left|\boldsymbol{p}+\boldsymbol{p}_{0}-\boldsymbol{p}_{1}\right| \boldsymbol{p}_{0}\right)\right) \\
& +W_{2}\left(\boldsymbol{p}-\boldsymbol{p}_{1}\right) W_{1}\left(\boldsymbol{p}_{1}-\boldsymbol{p}_{0}\right) \overline{\boldsymbol{X}}^{(11) 21}\left(\boldsymbol{p}\left|\boldsymbol{p}_{1}\right| \boldsymbol{p}_{0}\right) \\
& \left.\odot\left(\overline{\boldsymbol{X}}^{(11) 21}\left(\boldsymbol{p}\left|\boldsymbol{p}_{1}\right| \boldsymbol{p}_{0}\right)+\overline{\boldsymbol{X}}^{(11) 12}\left(\boldsymbol{p}\left|\boldsymbol{p}+\boldsymbol{p}_{0}-\boldsymbol{p}_{1}\right| \boldsymbol{p}_{0}\right)\right)\right]
\end{aligned}
$$

with

$$
\begin{aligned}
& \overline{\boldsymbol{X}}^{(30)}\left(\boldsymbol{p} \mid \boldsymbol{p}_{0}\right)=\int \frac{\mathrm{d}^{2} \boldsymbol{p}_{1}}{(2 \pi)^{2}}\left[W_{1}\left(\boldsymbol{p}_{1}-\boldsymbol{p}_{0}\right) \overline{\boldsymbol{X}}^{(30)}\left(\boldsymbol{p}\left|\boldsymbol{p}_{0}\right| \boldsymbol{p}_{1} \mid \boldsymbol{p}_{0}\right)\right. \\
& \left.+W_{1}\left(\boldsymbol{p}-\boldsymbol{p}_{1}\right)\left(\overline{\boldsymbol{X}}^{(30)}\left(\boldsymbol{p}\left|\boldsymbol{p}_{1}\right| \boldsymbol{p}_{0}-\boldsymbol{p}+\boldsymbol{p}_{1} \mid \boldsymbol{p}_{0}\right)+\overline{\boldsymbol{X}}^{(30)}\left(\boldsymbol{p}\left|\boldsymbol{p}_{1}\right| \boldsymbol{p} \mid \boldsymbol{p}_{0}\right)\right)\right], \\
& \overline{\boldsymbol{X}}^{(03)}\left(\boldsymbol{p} \mid \boldsymbol{p}_{0}\right)=\int \frac{\mathrm{d}^{2} \boldsymbol{p}_{1}}{(2 \pi)^{2}}\left[W_{2}\left(\boldsymbol{p}_{1}-\boldsymbol{p}_{0}\right) \overline{\boldsymbol{X}}^{(03)}\left(\boldsymbol{p}\left|\boldsymbol{p}_{0}\right| \boldsymbol{p}_{1} \mid \boldsymbol{p}_{0}\right)\right. \\
& \left.+W_{2}\left(\boldsymbol{p}-\boldsymbol{p}_{1}\right)\left(\overline{\boldsymbol{X}}^{(03)}\left(\boldsymbol{p}\left|\boldsymbol{p}_{1}\right| \boldsymbol{p}_{0}-\boldsymbol{p}+\boldsymbol{p}_{1} \mid \boldsymbol{p}_{0}\right)+\overline{\boldsymbol{X}}^{(03)}\left(\boldsymbol{p}\left|\boldsymbol{p}_{1}\right| \boldsymbol{p} \mid \boldsymbol{p}_{0}\right)\right)\right], \\
& \overline{\boldsymbol{X}}^{(12)}\left(\boldsymbol{p} \mid \boldsymbol{p}_{0}\right)=\int \frac{\mathrm{d}^{2} \boldsymbol{p}_{1}}{(2 \pi)^{2}}\left[W_{2}\left(\boldsymbol{p}-\boldsymbol{p}_{1}\right)\left(\overline{\boldsymbol{X}}^{(12) 221}\left(\boldsymbol{p}\left|\boldsymbol{p}_{1}\right| \boldsymbol{p} \mid \boldsymbol{p}_{0}\right)+\overline{\boldsymbol{X}}^{(12) 212}\left(\boldsymbol{p}\left|\boldsymbol{p}_{1}\right| \boldsymbol{p}_{0}-\boldsymbol{p}+\boldsymbol{p}_{1} \mid \boldsymbol{p}_{0}\right)\right)\right. \\
& \left.+W_{2}\left(\boldsymbol{p}_{1}-\boldsymbol{p}_{0}\right) \overline{\boldsymbol{X}}^{(12) 122}\left(\boldsymbol{p}\left|\boldsymbol{p}_{0}\right| \boldsymbol{p}_{1} \mid \boldsymbol{p}_{0}\right)\right], \\
& \overline{\boldsymbol{X}}^{(21)}\left(\boldsymbol{p} \mid \boldsymbol{p}_{0}\right)=\int \frac{\mathrm{d}^{2} \boldsymbol{p}_{1}}{(2 \pi)^{2}}\left[W_{1}\left(\boldsymbol{p}-\boldsymbol{p}_{1}\right)\left(\overline{\boldsymbol{X}}^{(21) 112}\left(\boldsymbol{p}\left|\boldsymbol{p}_{1}\right| \boldsymbol{p} \mid \boldsymbol{p}_{0}\right)+\overline{\boldsymbol{X}}^{(21) 121}\left(\boldsymbol{p}\left|\boldsymbol{p}_{1}\right| \boldsymbol{p}_{0}-\boldsymbol{p}+\boldsymbol{p}_{1} \mid \boldsymbol{p}_{0}\right)\right)\right. \\
& \left.+W_{1}\left(\boldsymbol{p}_{1}-\boldsymbol{p}_{0}\right) \overline{\boldsymbol{X}}^{(21) 211}\left(\boldsymbol{p}\left|\boldsymbol{p}_{0}\right| \boldsymbol{p}_{1} \mid \boldsymbol{p}_{0}\right)\right],
\end{aligned}
$$

\section{PERTURBATIVE DEVELOPMENT AND REDUCED RAYLEIGH EQUATIONS}

In order to obtain the development 24 for the scattering matrix, a practical method is provided by the use of the refuced Rayleigh equations first obtained by Brown et al $\mathbf{6}$ for a single rough surface, and then extended in $\mathbf{6}$ for a more general system. These equations are exact, under the Rayleigh hypothesis, and their main advantages are that one of the electric fields $\boldsymbol{E}^{i}, \boldsymbol{E}^{s}, \boldsymbol{E}^{1-}, \boldsymbol{E}^{1+}$ (see Fig. 2) of the problem has been eliminated. These equations are derived by taking linear combinations of the electromagnetic boundaries conditions at the first surface $h_{1}$, where the Fourier transform of the fields has been introduced. In particular, we obtain the two following equations (see Eqs.(99-100) in 9 ):

$$
\begin{gathered}
\int \frac{\mathrm{d}^{2} \boldsymbol{p}}{(2 \pi)^{2}} \overline{\boldsymbol{M}}_{h}^{1+, 0+}(\boldsymbol{u} \mid \boldsymbol{p}) \cdot \overline{\boldsymbol{R}}\left(\boldsymbol{p} \mid \boldsymbol{p}_{0}\right) \cdot \boldsymbol{E}^{i}\left(\boldsymbol{p}_{0}\right)+\overline{\boldsymbol{M}}_{h}^{1+, 0-}\left(\boldsymbol{u} \mid \boldsymbol{p}_{0}\right) \cdot \boldsymbol{E}^{i}\left(\boldsymbol{p}_{0}\right)=\frac{2\left(\epsilon_{0} \epsilon_{1}\right)^{\frac{1}{2}} \alpha_{1}(\boldsymbol{u})}{\left(\epsilon_{1}-\epsilon_{0}\right)} \boldsymbol{E}^{1+}(\boldsymbol{u}) \\
\int \frac{\mathrm{d}^{2} \boldsymbol{p}}{(2 \pi)^{2}} \overline{\boldsymbol{M}}_{h}^{1-, 0+}(\boldsymbol{u} \mid \boldsymbol{p}) \cdot \overline{\boldsymbol{R}}\left(\boldsymbol{p} \mid \boldsymbol{p}_{0}\right) \cdot \boldsymbol{E}^{i}\left(\boldsymbol{p}_{0}\right)+\overline{\boldsymbol{M}}_{h}^{1-, 0-}\left(\boldsymbol{u} \mid \boldsymbol{p}_{0}\right) \cdot \boldsymbol{E}^{i}\left(\boldsymbol{p}_{0}\right)=-\frac{2\left(\epsilon_{0} \epsilon_{1}\right)^{\frac{1}{2}} \alpha_{1}(\boldsymbol{u})}{\left(\epsilon_{1}-\epsilon_{0}\right)} \boldsymbol{E}^{1-}(\boldsymbol{u})
\end{gathered}
$$

where

$$
\overline{\boldsymbol{M}}_{h}^{1 b, 0 a}(\boldsymbol{u} \mid \boldsymbol{p}) \equiv \frac{I\left(b \alpha_{1}(\boldsymbol{u})-a \alpha_{0}(\boldsymbol{p}) \mid \boldsymbol{u}-\boldsymbol{p}\right)}{b \alpha_{1}(\boldsymbol{u})-a \alpha_{0}(\boldsymbol{p})} \overline{\boldsymbol{M}}^{1 b, 0 a}(\boldsymbol{u} \mid \boldsymbol{p})
$$

with

$$
\overline{\boldsymbol{M}}^{1 b, 0 a}(\boldsymbol{u} \mid \boldsymbol{p})=\left(\begin{array}{cc}
\|\boldsymbol{u}\|\|\boldsymbol{p}\|+a b \alpha_{1}(\boldsymbol{u}) \alpha_{0}(\boldsymbol{p}) \hat{\boldsymbol{u}} \cdot \hat{\boldsymbol{p}} & -b \epsilon_{0}^{\frac{1}{2}} K_{0} \alpha_{1}(\boldsymbol{u})(\hat{\boldsymbol{u}} \times \hat{\boldsymbol{p}})_{z} \\
a \epsilon_{1}^{\frac{1}{2}} K_{0} \alpha_{0}(\boldsymbol{p})(\hat{\boldsymbol{u}} \times \hat{\boldsymbol{p}})_{z} & \left(\epsilon_{0} \epsilon_{1}\right)^{\frac{1}{2}} K_{0}^{2} \hat{\boldsymbol{u}} \cdot \hat{\boldsymbol{p}}
\end{array}\right)
$$


and

$$
I(\alpha \mid \boldsymbol{p}) \equiv \int \mathrm{d}^{2} \boldsymbol{x} \exp \left(-\mathrm{i} \boldsymbol{p} \cdot \boldsymbol{x}-i \alpha h_{1}(\boldsymbol{x})\right)
$$

the symbols $a= \pm$ and $b= \pm$ in Eqs. (59 60 ) represent a given choice linked to the field propagation. In order to obtain a single equation for $\boldsymbol{R}\left(\boldsymbol{p} \mid \boldsymbol{p}_{0}\right)$, we have to find a relation between $\boldsymbol{E}^{1-}$ and $\boldsymbol{E}^{1+}$. To this end we already know an expression of the scattering matrix for a single rough surface separating two homogenous media of permittivity $\epsilon_{1}$ and $\epsilon_{2}$, which is translated along the $z$-axis to the height $z=-H$, and illuminated by a plane wave $E^{1-}(\boldsymbol{p})$. This scattering matrix denoted $\overline{\boldsymbol{R}}_{s \epsilon_{1}, \epsilon_{2}}^{H}$ is given by:

$$
\overline{\boldsymbol{R}}_{s \epsilon_{1}, \epsilon_{2}}^{H}\left(\boldsymbol{p} \mid \boldsymbol{p}_{0}\right)=\exp \left(\mathrm{i}\left(\alpha_{1}(\boldsymbol{p})+\alpha_{1}\left(\boldsymbol{p}_{0}\right)\right) H\right) \overline{\boldsymbol{R}}_{s \epsilon_{1}, \epsilon_{2}}\left(\boldsymbol{p} \mid \boldsymbol{p}_{0}\right),
$$

where $\overline{\boldsymbol{R}}_{s \epsilon_{1}, \epsilon_{2}}\left(\boldsymbol{p} \mid \boldsymbol{p}_{0}\right)$ can be found in Refs. $z=-H$ of the rough surface $h_{2}(\boldsymbol{x})$ (see Refs. 1 ). Thus we have the following relation:

$$
\boldsymbol{E}^{1+}(\boldsymbol{u})=\int \frac{\mathrm{d}^{2} \boldsymbol{u}_{1}}{(2 \pi)^{2}} \overline{\boldsymbol{R}}_{s \epsilon_{1}, \epsilon_{2}}^{H}\left(\boldsymbol{u} \mid \boldsymbol{u}_{1}\right) \cdot \boldsymbol{E}^{1-}\left(\boldsymbol{u}_{1}\right)
$$

Now combining Eq. (62) with Eqs. (56 55 ), we obtain an integral equation where $\overline{\boldsymbol{R}}\left(\boldsymbol{p} \mid \boldsymbol{p}_{0}\right)$ is the only unknown:

$$
\begin{aligned}
\int \frac{\mathrm{d}^{2} \boldsymbol{p}}{(2 \pi)^{2}} & {\left[\overline{\boldsymbol{M}}_{h}^{1+, 0+}(\boldsymbol{u} \mid \boldsymbol{p})+\int \frac{\mathrm{d}^{2} \boldsymbol{u}_{1}}{(2 \pi)^{2}} \frac{\alpha_{1}(\boldsymbol{u})}{\alpha_{1}\left(\boldsymbol{u}_{1}\right)} \overline{\boldsymbol{R}}_{s \epsilon_{1}, \epsilon_{2}}^{H}\left(\boldsymbol{u} \mid \boldsymbol{u}_{1}\right) \cdot \overline{\boldsymbol{M}}_{h}^{1-, 0+}\left(\boldsymbol{u}_{1} \mid \boldsymbol{p}\right)\right] \cdot \overline{\boldsymbol{R}}\left(\boldsymbol{p} \mid \boldsymbol{p}_{0}\right)=} \\
& -\left[\overline{\boldsymbol{M}}_{h}^{1+, 0-}\left(\boldsymbol{u} \mid \boldsymbol{p}_{0}\right)+\int \frac{\mathrm{d}^{2} \boldsymbol{u}_{1}}{(2 \pi)^{2}} \frac{\alpha_{1}(\boldsymbol{u})}{\alpha_{1}\left(\boldsymbol{u}_{1}\right)} \overline{\boldsymbol{R}}_{s \epsilon_{1}, \epsilon_{2}}^{H}\left(\boldsymbol{u} \mid \boldsymbol{u}_{1}\right) \cdot \overline{\boldsymbol{M}}_{h}^{1-, 0-}\left(\boldsymbol{u} \mid \boldsymbol{p}_{0}\right)\right] .
\end{aligned}
$$

Expanding $I(\alpha \mid \boldsymbol{p})$ in Eq. (60) in power of $h_{1}$ :

$$
\begin{aligned}
I(\alpha \mid \boldsymbol{p}) & =(2 \pi)^{2} \delta(\boldsymbol{p})-i \alpha h_{1}^{(1)}(\boldsymbol{p})-\frac{\alpha^{2}}{2} h_{1}^{(2)}(\boldsymbol{p})-\frac{i \alpha^{3}}{3 !} h_{1}^{(3)}(\boldsymbol{p})+\cdots, \\
h_{1}^{(n)}(\boldsymbol{p}) & \equiv \int \mathrm{d}^{2} \boldsymbol{x} \exp (-i \boldsymbol{p} \cdot \boldsymbol{x}) h_{1}^{n}(\boldsymbol{x}),
\end{aligned}
$$

and using the perturbative development 9 of $\overline{\boldsymbol{R}}_{s \epsilon_{1}, \epsilon_{2}}^{H}$ in power of $h_{2}$ :

$$
\begin{aligned}
\overline{\boldsymbol{R}}_{s \epsilon_{1}, \epsilon_{2}}^{H}\left(\boldsymbol{p} \mid \boldsymbol{p}_{0}\right)= & (2 \pi)^{2} \delta\left(\boldsymbol{p}-\boldsymbol{p}_{0}\right) \overline{\boldsymbol{X}}_{s \epsilon_{1}, \epsilon_{2}}^{H(0)}\left(\boldsymbol{p}_{0}\right)+\alpha_{0}\left(\boldsymbol{p}_{0}\right) \overline{\boldsymbol{X}}_{s \epsilon_{1}, \epsilon_{2}}^{H(1)}\left(\boldsymbol{p} \mid \boldsymbol{p}_{0}\right) h_{2}\left(\boldsymbol{p}-\boldsymbol{p}_{0}\right) \\
& +\alpha_{0}\left(\boldsymbol{p}_{0}\right) \int \frac{\mathrm{d}^{2} \boldsymbol{p}_{1}}{(2 \pi)^{2}} \overline{\boldsymbol{X}}_{s(2)}^{H\left(\epsilon_{1}, \epsilon_{2}\right.}\left(\boldsymbol{p}\left|\boldsymbol{p}_{1}\right| \boldsymbol{p}_{0}\right) h_{2}\left(\boldsymbol{p}-\boldsymbol{p}_{1}\right) h_{2}\left(\boldsymbol{p}_{1}-\boldsymbol{p}_{0}\right) \\
& +\alpha_{0}\left(\boldsymbol{p}_{0}\right) \iint \frac{\mathrm{d}^{2} \boldsymbol{p}_{1}}{(2 \pi)^{2}} \frac{\mathrm{d}^{2} \boldsymbol{p}_{2}}{(2 \pi)^{2}} \overline{\boldsymbol{X}}_{s \epsilon_{1}, \epsilon_{2}}^{H(3)}\left(\boldsymbol{p}\left|\boldsymbol{p}_{1}\right| \boldsymbol{p}_{2} \mid \boldsymbol{p}_{0}\right) h_{2}\left(\boldsymbol{p}-\boldsymbol{p}_{1}\right) h_{2}\left(\boldsymbol{p}_{1}-\boldsymbol{p}_{2}\right) h_{2}\left(\boldsymbol{p}_{2}-\boldsymbol{p}_{0}\right),
\end{aligned}
$$

we finally obtain the expansions 24, 31-39). The expression for the scattering matrix when only one rough surface is involved was given in Ref. 9 , here a small change have been made in the notations:

$$
\begin{gathered}
\overline{\boldsymbol{R}}^{(n 0)}\left(\boldsymbol{p} \mid \boldsymbol{p}_{0}\right)=\overline{\boldsymbol{R}}_{u}^{(n)}\left(\boldsymbol{p} \mid \boldsymbol{p}_{0}\right), \\
\overline{\boldsymbol{R}}^{(0 n)}\left(\boldsymbol{p} \mid \boldsymbol{p}_{0}\right)=\overline{\boldsymbol{R}}_{d}^{(n)}\left(\boldsymbol{p} \mid \boldsymbol{p}_{0}\right)
\end{gathered}
$$


$n$ being an integer ranging from 0 to 3 . For the others coefficients we have :

$$
\begin{aligned}
& \overline{\boldsymbol{X}}^{(11) 21}\left(\boldsymbol{p}\left|\boldsymbol{p}_{1}\right| \boldsymbol{p}_{0}\right)=\overline{\boldsymbol{T}}^{10}(\boldsymbol{p}) \cdot \overline{\boldsymbol{U}}^{(0)}(\boldsymbol{p}) \cdot \overline{\boldsymbol{X}}_{s \epsilon_{1}, \epsilon_{2}}^{H(1)}\left(\boldsymbol{p} \mid \boldsymbol{p}_{1}\right) \cdot\left[-\overline{\boldsymbol{\epsilon}} \cdot \overline{\boldsymbol{D}}_{10}^{-}\left(\boldsymbol{p}_{1}\right) \cdot \overline{\boldsymbol{X}}^{(10)}\left(\boldsymbol{p}_{1} \mid \boldsymbol{p}_{0}\right)+i \overline{\boldsymbol{S}}^{+}\left(\boldsymbol{p}_{1} \mid \boldsymbol{p}_{0}\right)\right] \\
& \overline{\boldsymbol{X}}^{(11) 12}\left(\boldsymbol{p}\left|\boldsymbol{p}_{1}\right| \boldsymbol{p}_{0}\right)=\mathrm{i} \overline{\boldsymbol{P}}^{+}\left(\boldsymbol{p} \mid \boldsymbol{p}_{1}\right) \cdot \overline{\boldsymbol{X}}^{(01)}\left(\boldsymbol{p}_{1} \mid \boldsymbol{p}_{0}\right), \\
& \overline{\boldsymbol{X}}^{(21) 112}\left(\boldsymbol{p}\left|\boldsymbol{p}_{1}\right| \boldsymbol{p}_{2} \mid \boldsymbol{p}_{0}\right)=\mathrm{i} \overline{\boldsymbol{P}}^{+}\left(\boldsymbol{p} \mid \boldsymbol{p}_{1}\right) \cdot \overline{\boldsymbol{X}}^{(11) 12}\left(\boldsymbol{p}_{1}\left|\boldsymbol{p}_{2}\right| \boldsymbol{p}_{0}\right) \\
& +\frac{1}{2}\left[\alpha_{1}(\boldsymbol{p}) \overline{\boldsymbol{P}}^{-}\left(\boldsymbol{p} \mid \boldsymbol{p}_{2}\right)-\alpha_{0}\left(\boldsymbol{p}_{2}\right) \overline{\boldsymbol{P}}^{+}\left(\boldsymbol{p} \mid \boldsymbol{p}_{2}\right)\right] \cdot \overline{\boldsymbol{X}}^{(01)}\left(\boldsymbol{p}_{2} \mid \boldsymbol{p}_{0}\right) \\
& \overline{\boldsymbol{X}}^{(21) 121}\left(\boldsymbol{p}\left|\boldsymbol{p}_{1}\right| \boldsymbol{p}_{2} \mid \boldsymbol{p}_{0}\right)=\mathrm{i} \overline{\boldsymbol{P}}^{+}\left(\boldsymbol{p} \mid \boldsymbol{p}_{1}\right) \cdot \overline{\boldsymbol{X}}^{(11) 21}\left(\boldsymbol{p}_{1}\left|\boldsymbol{p}_{2}\right| \boldsymbol{p}_{0}\right), \\
& \overline{\boldsymbol{X}}^{(21) 211}\left(\boldsymbol{p}\left|\boldsymbol{p}_{1}\right| \boldsymbol{p}_{2} \mid \boldsymbol{p}_{0}\right)=\overline{\boldsymbol{T}}^{10}(\boldsymbol{p}) \cdot \overline{\boldsymbol{U}}^{(0)}(\boldsymbol{p}) \cdot \overline{\boldsymbol{X}}_{\boldsymbol{s} \epsilon_{1}, \epsilon_{2}}^{H(1)}\left(\boldsymbol{p} \mid \boldsymbol{p}_{1}\right) \cdot\left[-\overline{\boldsymbol{\epsilon}} \cdot \overline{\boldsymbol{D}}_{10}^{-}\left(\boldsymbol{p}_{1}\right) \cdot \overline{\boldsymbol{X}}^{(20)}\left(\boldsymbol{p}_{1}\left|\boldsymbol{p}_{2}\right| \boldsymbol{p}_{0}\right)\right. \\
& +\frac{i\left(\epsilon_{1}-\epsilon_{0}\right)}{2\left(\epsilon_{0} \epsilon_{1}\right)^{1 / 2}} \overline{\boldsymbol{M}}^{1-, 0+}\left(\boldsymbol{p}_{1} \mid \boldsymbol{p}_{2}\right) \cdot \overline{\boldsymbol{X}}^{(10)}\left(\boldsymbol{p}_{2} \mid \boldsymbol{p}_{0}\right) \\
& \left.-\frac{1}{2}\left(\alpha_{1}\left(\boldsymbol{p}_{1}\right) \overline{\boldsymbol{S}}^{+}\left(\boldsymbol{p}_{1} \mid \boldsymbol{p}_{0}\right)+\alpha_{0}\left(\boldsymbol{p}_{0}\right) \overline{\boldsymbol{S}}^{-}\left(\boldsymbol{p}_{1} \mid \boldsymbol{p}_{0}\right)\right)\right] \\
& \overline{\boldsymbol{X}}^{(12) 122}\left(\boldsymbol{p}\left|\boldsymbol{p}_{1}\right| \boldsymbol{p}_{2} \mid \boldsymbol{p}_{0}\right)=\mathrm{i} \overline{\boldsymbol{P}}^{+}\left(\boldsymbol{p} \mid \boldsymbol{p}_{1}\right) \cdot \overline{\boldsymbol{X}}^{(02)}\left(\boldsymbol{p}_{1}\left|\boldsymbol{p}_{2}\right| \boldsymbol{p}_{0}\right), \\
& \overline{\boldsymbol{X}}^{(12) 212}\left(\boldsymbol{p}\left|\boldsymbol{p}_{1}\right| \boldsymbol{p}_{2} \mid \boldsymbol{p}_{0}\right)=\overline{\boldsymbol{T}}^{10}(\boldsymbol{p}) \cdot \overline{\boldsymbol{U}}^{(0)}(\boldsymbol{p}) \cdot \overline{\boldsymbol{X}}_{s \epsilon_{1}, \epsilon_{2}}^{H(1)}\left(\boldsymbol{p} \mid \boldsymbol{p}_{1}\right) \cdot\left[-\overline{\boldsymbol{\epsilon}} \cdot \overline{\boldsymbol{D}}_{10}^{-}\left(\boldsymbol{p}_{1}\right) \cdot \overline{\boldsymbol{X}}^{(11) 12}\left(\boldsymbol{p}_{1}\left|\boldsymbol{p}_{2}\right| \boldsymbol{p}_{0}\right)\right. \\
& \left.+\frac{i\left(\epsilon_{1}-\epsilon_{0}\right)}{2\left(\epsilon_{0} \epsilon_{1}\right)^{1 / 2}} \overline{\boldsymbol{M}}^{1-, 0+}\left(\boldsymbol{p}_{1} \mid \boldsymbol{p}_{2}\right) \cdot \overline{\boldsymbol{X}}^{(01)}\left(\boldsymbol{p}_{2} \mid \boldsymbol{p}_{0}\right)\right], \\
& \overline{\boldsymbol{X}}^{(12) 221}\left(\boldsymbol{p}\left|\boldsymbol{p}_{1}\right| \boldsymbol{p}_{2} \mid \boldsymbol{p}_{0}\right)=\overline{\boldsymbol{T}}^{10}(\boldsymbol{p}) \cdot \overline{\boldsymbol{U}}^{(0)}(\boldsymbol{p}) \cdot\left[-\overline{\boldsymbol{X}}_{s \epsilon_{1}, \epsilon_{2}}^{H(1)}\left(\boldsymbol{p} \mid \boldsymbol{p}_{1}\right) \cdot \overline{\boldsymbol{\epsilon}} \cdot \overline{\boldsymbol{D}}_{10}^{-}\left(\boldsymbol{p}_{1}\right) \cdot \overline{\boldsymbol{X}}^{(11) 21}\left(\boldsymbol{p}_{1}\left|\boldsymbol{p}_{2}\right| \boldsymbol{p}_{0}\right)\right. \\
& \left.+\overline{\boldsymbol{X}}_{s \epsilon_{1}, \epsilon_{2}}^{H(2)}\left(\boldsymbol{p}\left|\boldsymbol{p}_{1}\right| \boldsymbol{p}_{2}\right) \cdot\left(-\overline{\boldsymbol{\epsilon}} \cdot \overline{\boldsymbol{D}}_{10}^{-}\left(\boldsymbol{p}_{2}\right) \cdot \overline{\boldsymbol{X}}^{(10)}\left(\boldsymbol{p}_{2} \mid \boldsymbol{p}_{0}\right)+\mathrm{i} \overline{\boldsymbol{S}}^{+}\left(\boldsymbol{p}_{2} \mid \boldsymbol{p}_{0}\right)\right)\right],
\end{aligned}
$$

where the matrices $\overline{\boldsymbol{T}}^{(10)}, \overline{\boldsymbol{U}}^{(0)}, \overline{\boldsymbol{P}}^{ \pm}, \overline{\boldsymbol{D}}_{10}^{ \pm}, \overline{\boldsymbol{X}}^{(10)}, \overline{\boldsymbol{X}}^{(20)}, \overline{\boldsymbol{X}}^{(01)}, \overline{\boldsymbol{X}}^{(02)}, \overline{\boldsymbol{X}}_{s \epsilon_{1}, \epsilon_{2}}^{H(1)}, \overline{\boldsymbol{X}}_{s \epsilon_{1}, \epsilon_{2}}^{H(2)}$ are respectively defined by Eqs.(71),(87),(114,115),(57),(104,105),(96,97),(60,61) in Ref. $\mathrm{Q}$, and $\overline{\boldsymbol{\epsilon}}, \overline{\boldsymbol{S}}^{ \pm}$are given in Appendix A.

\section{NUMERICAL RESULTS}

As an application of the previous formalism we consider a system made of a air-dielectric film whose dielectric constant is $\epsilon_{1}=2.6896+i 0.0075\left(\epsilon_{0}=1\right)$, deposited on silver surface with $\epsilon_{2}=-18.3+i 0.55$. The vacuum-dielectric interface is a two-dimensionnal rough surface, whose parameters are $\sigma_{1}=15 \mathrm{~nm}$ and $l_{1}=100 \mathrm{~nm}$. The dielectric-silver boundary is also rough and defined by $\sigma_{2}=5 \mathrm{~nm}$ and $l_{2}=100 \mathrm{~nm}$. The incident wave has an arbitrary polarization and his wavelength is $\lambda=632.8 \mathrm{pm}$. With this set of parameters, the conditions of validity of the small-perturbation theory are satisfied 20 namely:

$$
\begin{array}{rlrl}
2 \pi\left|\frac{\epsilon_{1}}{\epsilon_{0}}\right|^{1 / 2} \frac{\sigma_{1}}{\lambda} & \ll 1, \quad 2 \pi\left|\frac{\epsilon_{2}}{\epsilon_{1}}\right|^{1 / 2} \frac{\sigma_{2}}{\lambda} & \ll 1 \\
\frac{\sigma_{1}}{l_{1}} & \ll 1, & \frac{\sigma_{2}}{l_{2}} & \ll 1 .
\end{array}
$$

The thickness of the film is $H=500 \mathrm{~nm}$ and support two-guided wave polaritons for the (TE) polarizations at $p_{T E}^{1}=1.5534 K_{0}$, and $p_{T E}^{2}=1.2727 K_{0}$, and three guided-modes for the $(T M)$ polarizations at $p_{T M}^{1}=1.7752 K_{0}, p_{T M}^{2}=1.4577 K_{0}$ and $p_{T M}^{3}=1.034 K_{0}$. We have computed the incoherent bistatic coefficient $\gamma^{\text {incoh }}\left(\boldsymbol{p} \mid \boldsymbol{p}_{0}\right)$, where the integrals involved in Eqs. (44, 47, 51, 52 555) are evaluated using Legendre quadrature. The results are shown in Figs. (何), where the incoherent bistatic coefficient is drawn as of function of the scattering angle $\theta$ for two different angles of incidence and the incident wave is linearly polarized. In Fig. 4, the wave is normally incident and the scattered field is observed in the incident plane 
$\left(\phi=0^{\circ}\right)$. The single scattering contribution on each surface, associated with the terms $\overline{\boldsymbol{I}}^{(10-10)}+\overline{\boldsymbol{I}}^{(01-01)}$, is plotted as a dotted line, the double-scattering contribution $\overline{\boldsymbol{I}}^{(20-20)}+\overline{\boldsymbol{I}}^{(02-02)}+\overline{\boldsymbol{I}}^{(11-11)}$ as a dashed line, the other terms $\overline{\boldsymbol{I}}^{(30-10)}+\overline{\boldsymbol{I}}^{(03-01)}+\overline{\boldsymbol{I}}^{(12-10)}+\overline{\boldsymbol{I}}^{(21-01)}$ as a dash-dotted line, and the total contribution $\gamma^{\text {incoh }}$ by the solid curve. We observed an enhancement of the backscattering which corresponds to the physical process in which the incident light excites a guided-mode through the roughness of the slab and then is scattered into a volume wave which is also due to the roughness effect. During the same process, the light can follow this path in the opposite direction where one possible configuration is shown in Fig. 1. These two paths can interfere constructively near the backscattering direction to produce a peak. As these paths are identical for the two waves under consideration, they should have the same degree of interaction with the rough surface, thus a term like $\overline{\boldsymbol{I}}^{(30-10)}$ cannot produce the peak because the first wave interact three times with the upper rough surface while the second wave only one time. The effect comes only from the terms $\overline{\boldsymbol{I}}^{(20-20)}+\overline{\boldsymbol{I}}^{(02-02)}+\overline{\boldsymbol{I}}^{(11-11)}$, which contain the paths indicated in Fig. 1. However, it has to be noticed that these terms contain also paths that do not produce enhanced backscattering, for example in $\overline{\boldsymbol{I}}^{(20-20)}$ we have the scattering process where the incident wave is only scattered one time by the upper rough surface but the scattering process is of order two in $h_{1}$. This is the reason why the terms $\overline{\boldsymbol{I}}^{(20-20)}+\overline{\boldsymbol{I}}^{(02-02)}+\overline{\boldsymbol{I}}^{(11-11)}$ are not zero away from the anti-specular direction. In order to separate the different contributions to the backscattering peak, we have drawn the contributions of $\overline{\boldsymbol{I}}^{(20-20)}, \overline{\boldsymbol{I}}^{(02-02)}, \overline{\boldsymbol{I}}^{(11-11)}$ separately in Fig. 目 as a dashed-line, dotted-line and solid curve respectively, we see that each term produces an enhancement near the anti-specular direction. The terms $\overline{\boldsymbol{I}}^{(20-20)}, \overline{\boldsymbol{I}}^{(02-02)}$ are the classic one(see Refs 9.15$)$ where the fields do not interact with both rough surfaces but produce the peak due to the scattering on the same rough surface: the upper one for $\overline{\boldsymbol{I}}^{(20-20)}$ and the bottom one for $\overline{\boldsymbol{I}}^{(02-02)}$. The contribution $\overline{\boldsymbol{I}}^{(11-11)}$ is the new result of this work, because we see that the mechanism of Fig. 1 exists and has the same magnitude as the other terms for the choosen parameters. In order to clearly show the displacement of the backscattering enhancement as the angle of incidence is varied, we show in Figs. 6 and 7 the numerical results of the perturbation method when $\theta_{0}=20^{\circ}$. As expected, we clearly see the peak which is now located at $\theta_{0}=-20^{\circ}$. Recent papers 15 have also explored the satellite peaks phenomenon which occurs when the wave follows two reverse paths

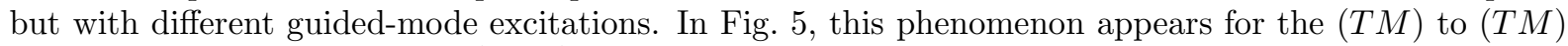
polarization due to the term $\overline{\boldsymbol{I}}^{(02-02)}$. Although a similar phenomenon occurs for the other terms it is too weak to contribute significantly..

\section{CONCLUSION}

In conclusion, the results in this paper clearly show that the backscattering enhancement produced by a rough slab due to the guided-wave polaritons has several origin. At small order in the perturbative development, the peak is produced by a double scattering mechanism. The new results we would like to emphasize, is that not only processes where the two scattering events take place on the same rough surface are capable to produce the backscattering peak, but it is also due to the fact that the wave can be scattered first by one of the rough surface and second by the other surface. To carry the proof, we have performed a perturbative development up to order four in the rms-height of the surfaces which has been possible using an integral equation called reduced Rayleigh equation, in which only the scattering matrix of the whole structure is unknown.

\section{ACKNOWLEDGMENTS}

(AS) thanks ANRT for financial support during the preparation of his thesis (contract CIFRE-238-98). 


\section{APPENDIX A: SCATTERING MATRIX COEFFICIENTS}

Here we collect the expression of the matrices not given in 9 :

$$
\begin{aligned}
\overline{\boldsymbol{\epsilon}} & \equiv \frac{1}{2}\left(\begin{array}{cc}
\left(\epsilon_{0} \epsilon_{1}\right)^{-1 / 2} & 0 \\
0 & 1
\end{array}\right) \\
\overline{\boldsymbol{S}}^{ \pm}\left(\boldsymbol{p} \mid \boldsymbol{p}_{0}\right) & \equiv \frac{\epsilon_{1}-\epsilon_{0}}{2 \alpha_{0}\left(\boldsymbol{p}_{0}\right)\left(\epsilon_{0} \epsilon_{1}\right)^{1 / 2}}\left[\overline{\boldsymbol{M}}^{1-, 0+}\left(\boldsymbol{p} \mid \boldsymbol{p}_{0}\right) . \cdot \overline{\boldsymbol{X}}^{(00)}\left(\boldsymbol{p} \mid \boldsymbol{p}_{0}\right) \pm \overline{\boldsymbol{M}}^{1-, 0-}\left(\boldsymbol{p} \mid \boldsymbol{p}_{0}\right)\right] .
\end{aligned}
$$

After some calculations we obtain :

$$
\begin{aligned}
& \overline{\boldsymbol{S}}^{+}\left(\boldsymbol{p} \mid \boldsymbol{p}_{0}\right)=\frac{\left(\epsilon_{1}-\epsilon_{0}\right)}{\left(\epsilon_{0} \epsilon_{1}\right)^{1 / 2}} \\
& \times\left(\begin{array}{cc}
\epsilon_{1}\|\boldsymbol{p}\|\left\|\boldsymbol{p}_{0}\right\| F_{V}^{+}\left(\boldsymbol{p}_{0}\right)+\epsilon_{0} \alpha_{1}(\boldsymbol{p}) \alpha_{1}\left(\boldsymbol{p}_{0}\right) F_{V}^{-}\left(\boldsymbol{p}_{0}\right) \hat{\boldsymbol{p}} \cdot \hat{\boldsymbol{p}}_{0} & \epsilon_{0}^{1 / 2} K_{0} \alpha_{1}(\boldsymbol{p}) F_{H}^{+}\left(\boldsymbol{p}_{0}\right)\left(\hat{\boldsymbol{p}} \times \hat{\boldsymbol{p}}_{0}\right)_{z} \\
-\epsilon_{0} \epsilon_{1}^{1 / 2} K_{0} \alpha_{1}\left(\boldsymbol{p}_{0}\right) F_{V}^{-}\left(\boldsymbol{p}_{0}\right)\left(\hat{\boldsymbol{p}} \times \hat{\boldsymbol{p}}_{0}\right)_{z} & \left(\epsilon_{0} \epsilon_{1}\right)^{1 / 2} K_{0}^{2} F_{H}^{+}\left(\boldsymbol{p}_{0}\right) \hat{\boldsymbol{p}} \cdot \hat{\boldsymbol{p}}_{0}
\end{array}\right) \\
& \text { · }\left[\overline{\boldsymbol{D}}_{10}^{+}\left(\boldsymbol{p}_{0}\right)\right]^{-1}, \\
& \overline{\boldsymbol{S}}^{-}\left(\boldsymbol{p} \mid \boldsymbol{p}_{0}\right)=\frac{\left(\epsilon_{1}-\epsilon_{0}\right)}{\alpha_{0}\left(\boldsymbol{p}_{0}\right)\left(\epsilon_{0} \epsilon_{1}\right)^{1 / 2}} \\
& \times\left(\begin{array}{cc}
-\epsilon_{0} \alpha_{1}\left(\boldsymbol{p}_{0}\right)\|\boldsymbol{p}\|\left\|\boldsymbol{p}_{0}\right\| F_{V}^{-}\left(\boldsymbol{p}_{0}\right) & -\epsilon_{0}^{1 / 2} K_{0} \alpha_{1}(\boldsymbol{p}) \alpha_{1}\left(\boldsymbol{p}_{0}\right) F_{H}^{-}\left(\boldsymbol{p}_{0}\right)\left(\hat{\boldsymbol{p}} \times \hat{\boldsymbol{p}}_{0}\right)_{z} \\
-\epsilon_{1} \alpha_{1}(\boldsymbol{p}) \alpha_{0}^{2}\left(\boldsymbol{p}_{0}\right) F_{V}^{+}\left(\boldsymbol{p}_{0}\right) \hat{\boldsymbol{p}} \cdot \hat{\boldsymbol{p}}_{0} & \\
\epsilon_{1}^{3 / 2} K_{0} \alpha_{0}^{2}\left(\boldsymbol{p}_{0}\right) F_{V}^{+}\left(\boldsymbol{p}_{0}\right)\left(\hat{\boldsymbol{p}} \times \hat{\boldsymbol{p}}_{0}\right)_{z} & -\left(\epsilon_{0} \epsilon_{1}\right)^{1 / 2} K_{0}^{2} \alpha_{1}\left(\boldsymbol{p}_{0}\right) F_{H}^{-}\left(\boldsymbol{p}_{0}\right) \hat{\boldsymbol{p}} \cdot \hat{\boldsymbol{p}}_{0}
\end{array}\right) \\
& \text {. }\left[\overline{\boldsymbol{D}}_{10}^{+}\left(\boldsymbol{p}_{0}\right)\right]^{-1} \text {. }
\end{aligned}
$$

\section{REFERENCES}

${ }^{1}$ I. Ohlí dal, K. Navrátil and M. Ohlí dal, "Scattering of light from multilayer systems with rough boundaries," Prog. Opt. 34, 251-334 (1995).

2 I. M. Fuks and A. G. Voronovich, "Wave diffraction by rough interfaces in an arbitrary plane-layered medium," Waves in Random Media 10, 253-272 (2000).

${ }^{3}$ R. García-Llamas, L. E. Regalado and C. Amra, "Sacttering of light from a two-layer system with a rough surface," J. Opt. Soc. Am. A 16, 2713-2719 (1999).

${ }^{4}$ P. Bousquet, F. Flory and P. Roche, "Scattering from multilayer thin films: theory and experiment," J. Opt. Soc. Am. A 71, 1115-23 (1981).

${ }^{5}$ J. M. Elson, "Multilayer-coated optics: guided-wave coupling and scattering of interface random roughness," J. Opt. Soc. Am. A 12, 729-742 (1995).

${ }^{6}$ S. O. Rice, "Reflection of electromagnetic waves from slightly rough surfaces," Comm. Pure Appl. Math. 4, 351-378 (1951).

7 A.G. Voronovich, Wave Scattering from Rough Surfaces (Springer, Berlin, 1994).

8 G. C. Brown, V. Celli, M. Haller and A. Marvin, "Vector theory of light scattering from a rough surface : unitary and reciprocal expansions," Surf. Sci. 136, 381-397 (1984).

9 A. Soubret, G. Berginc and C. Bourrely, "A new application of reduced Rayleigh equations to electromagnetic wave scattering by two-dimensional randomly rough surfaces", preprint CPT-2000/P.4016 cond-mat/0009329).

10 A. R. McGurn, A. A. Maradudin and V. Celli, "Localization effects in the scattering of light from a randomly rough grating," Phys. Rev. B 31, 4866-4871 (1985).

${ }^{11}$ V. Celli, A. A. Maradudin, A. M. Marvin and A. R. McGurn, "Some aspects of light scattering from a randomly rough metal surface," J. Opt. Soc. Am. A 2, 2225-2239 (1985).

12 A. R. McGurn and A. A. Maradudin, "Localization effects in the elastic scattering of light from a randomly rough surface," J. Opt. Soc. Am. B 4, 910-926 (1987).

13 A. A. Maradudin and E. R. Méndez, "Enhanced backscattering of light from weakly rough, random metal surfaces," Appl. Opt. 32, 3335-3343 (1993).

${ }^{14}$ C. S. West and K. A. O'Donnell "Observations of backscattering enhancement from polaritons on a rough metal surface," J. Opt. Soc. Am. A 12, 390-397 (1995). 
15 V. Freilikher, E. Kanzieper and A. A. Maradudin, "Coherent scattering enhancement in systems bounded by rough surfaces," Phys. Rep. 288, 127-204 (1997).

16 A. Ishimaru, C. Le, Y. Kuga, L. A. Sengers and T. K. Chan, "Polarimetric scattering theory for high slope rough surfaces," PIER 14, 1-36 (1996).

${ }^{17}$ L. Tsang, G. T. J. Kong and R. Shin, Theory of Microwave Remote Sensing (Wiley-Interscience, New York, 1985).

18 A. R. McGurn and A. A. Maradudin, "Perturbation theory results for the diffuse scattering of light from two-dimensional randomly rough metal surfaces," Waves in Random Media 6, 251-267 (1996).

19 J. T. Johnson, "Third-order small-perturbation method for scattering from dielectric rough surfaces," J. Opt. Soc. Am. A 16, 2720-2736 (1999).

20 J. A. Ogilvy, Theory of Wave Scattering From Random Rough Surfaces (Adam Hilger, Bristol, 1991).

\section{LIST OF FIGURES}

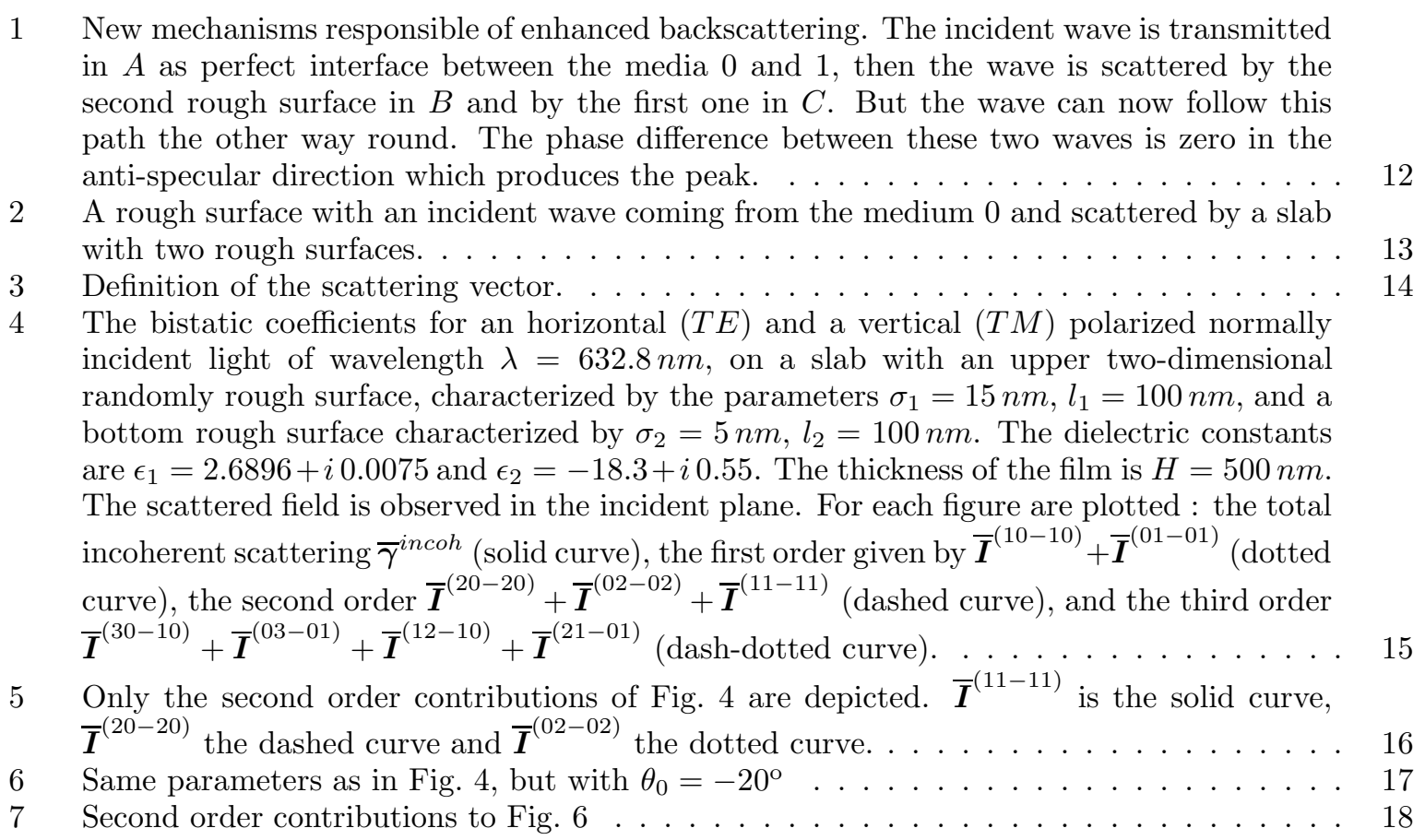




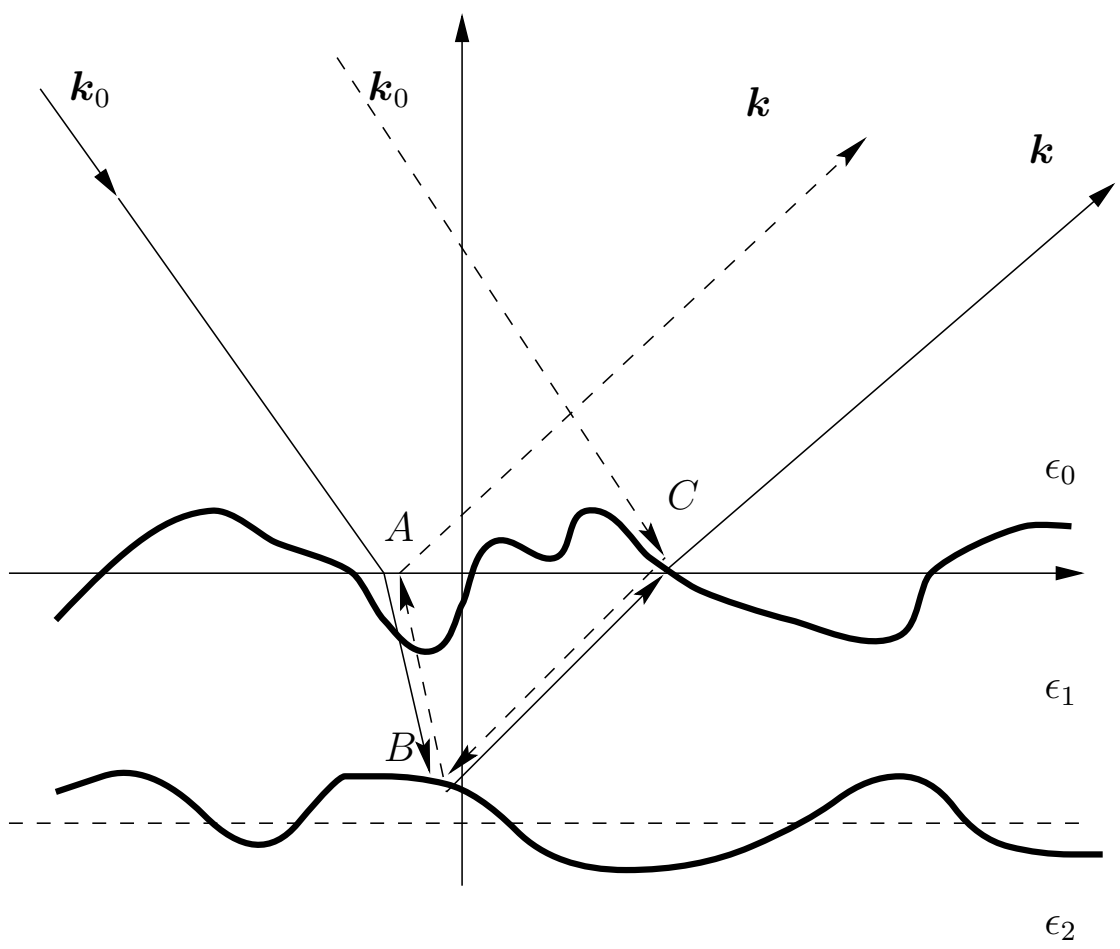

FIG. 1: New mechanisms responsible of enhanced backscattering. The incident wave is transmitted in $A$ as perfect interface between the media 0 and 1 , then the wave is scattered by the second rough surface in $B$ and by the first one in $C$. But the wave can now follow this path the other way round. The phase difference between these two waves is zero in the anti-specular direction which produces the peak. 


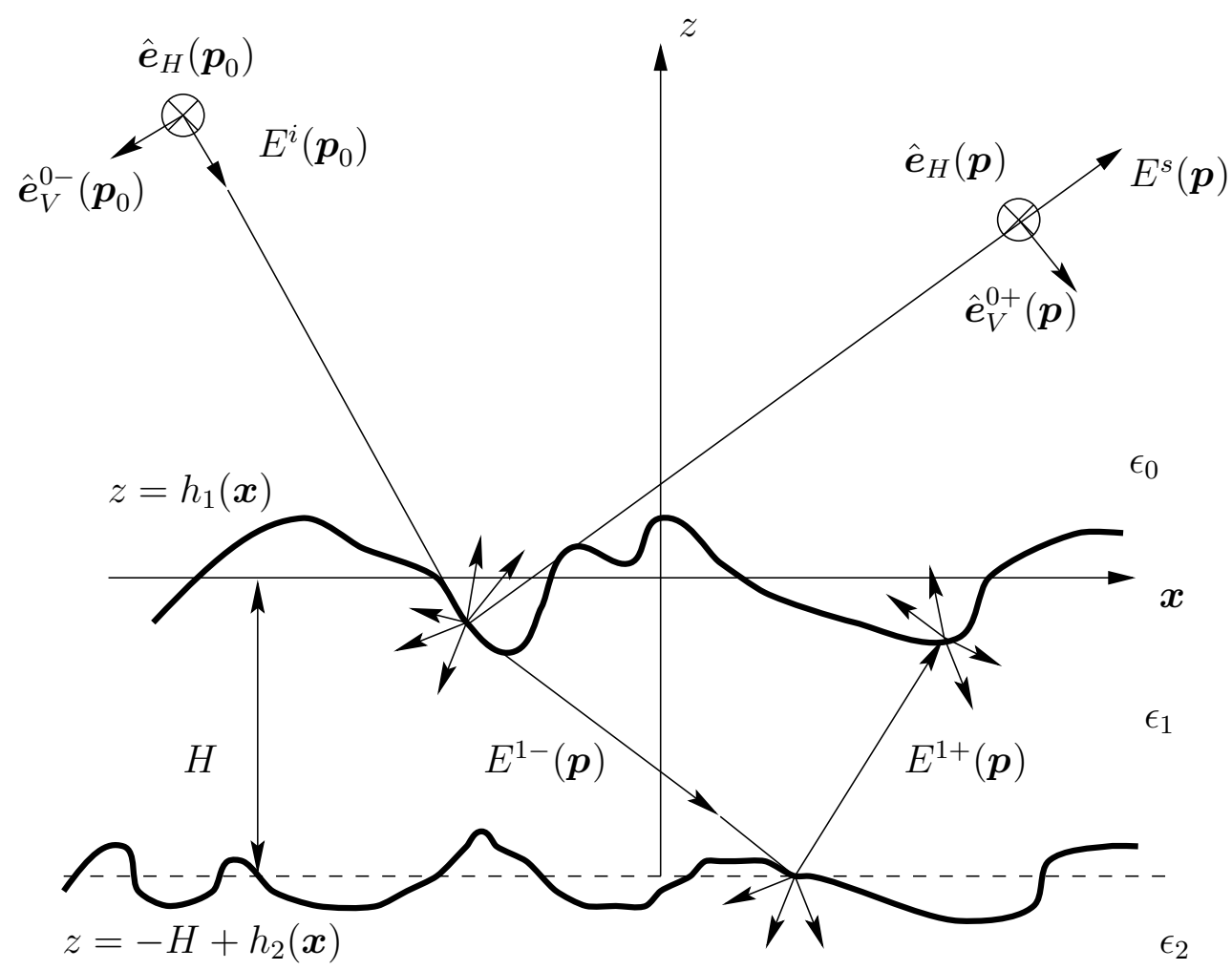

FIG. 2: A rough surface with an incident wave coming from the medium 0 and scattered by a slab with two rough surfaces. 


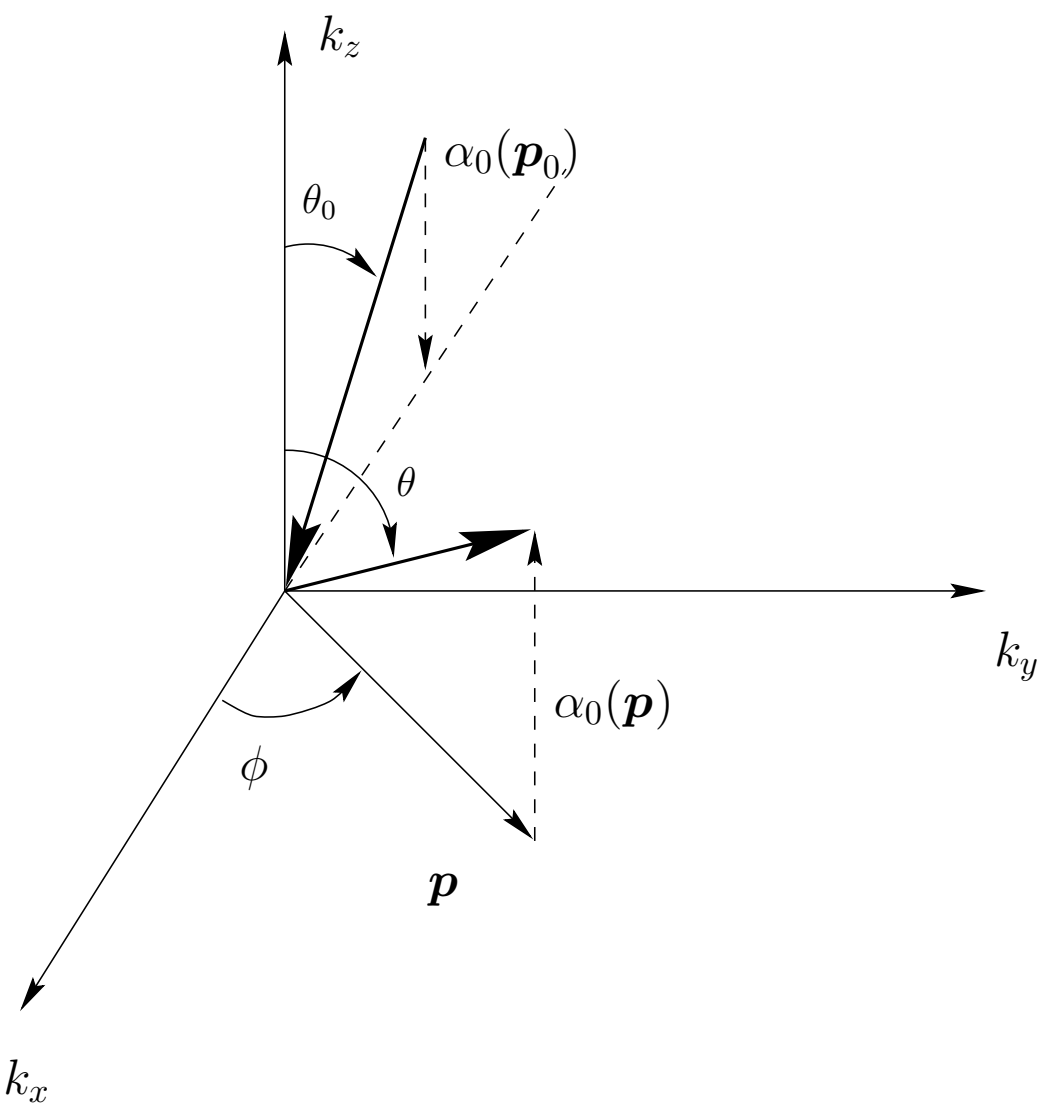

FIG. 3: Definition of the scattering vector. 

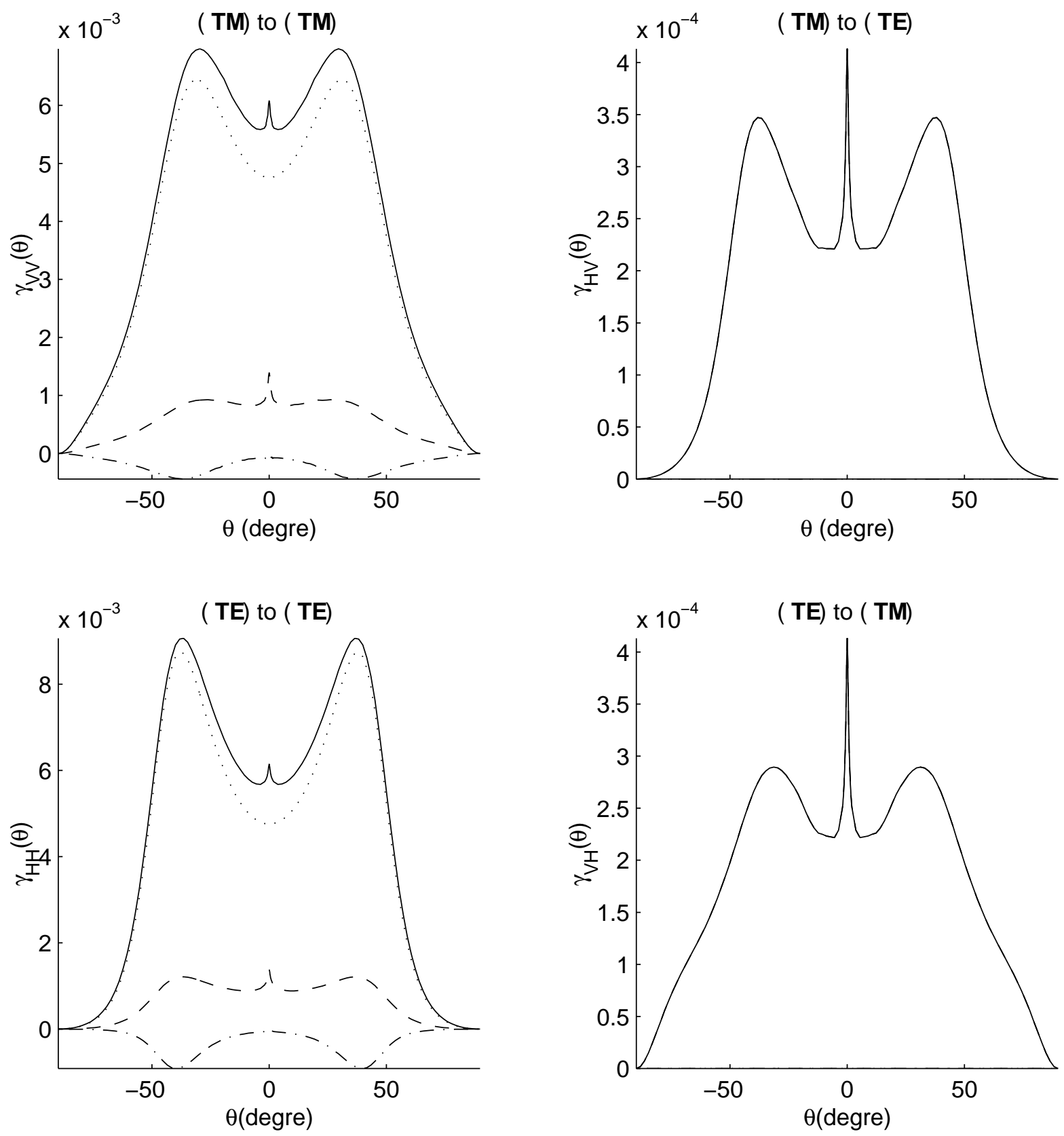

FIG. 4: The bistatic coefficients for an horizontal (TE) and a vertical $(T M)$ polarized normally incident light of wavelength $\lambda=632.8 \mathrm{~nm}$, on a slab with an upper two-dimensional randomly rough surface, characterized by the parameters $\sigma_{1}=15 \mathrm{~nm}, l_{1}=100 \mathrm{~nm}$, and a bottom rough surface characterized by $\sigma_{2}=5 \mathrm{~nm}, l_{2}=100 \mathrm{~nm}$. The dielectric constants are $\epsilon_{1}=2.6896+i 0.0075$ and $\epsilon_{2}=-18.3+i 0.55$. The thickness of the film is $H=500 \mathrm{~nm}$. The scattered field is observed in the incident plane. For each figure are plotted : the total incoherent scattering $\bar{\gamma}^{\text {incoh }}$ (solid curve), the first order given by $\overline{\boldsymbol{I}}^{(10-10)}+\overline{\boldsymbol{I}}^{(01-01)}$ (dotted curve), the second order $\overline{\boldsymbol{I}}^{(20-20)}+\overline{\boldsymbol{I}}^{(02-02)}+\overline{\boldsymbol{I}}^{(11-11)}$ (dashed curve), and the third order $\overline{\boldsymbol{I}}^{(30-10)}+\overline{\boldsymbol{I}}^{(03-01)}+\overline{\boldsymbol{I}}^{(12-10)}+\overline{\boldsymbol{I}}^{(21-01)}$ (dash-dotted curve). 

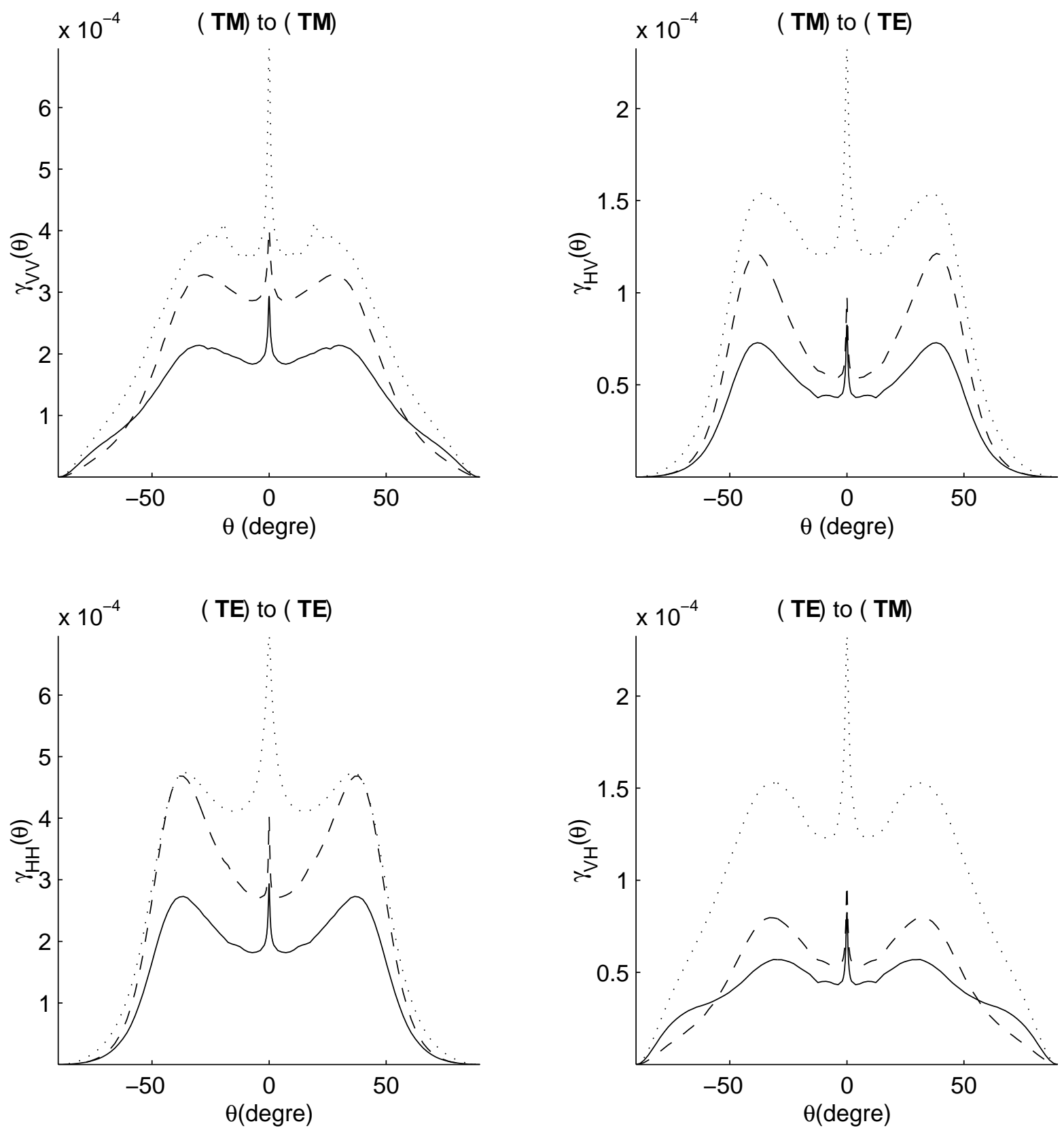

FIG. 5: Only the second order contributions of Fig. 0 are depicted. $\overline{\boldsymbol{I}}^{(11-11)}$ is the solid curve, $\overline{\boldsymbol{I}}^{(20-20)}$ the dashed curve and $\overline{\boldsymbol{I}}^{(02-02)}$ the dotted curve. 

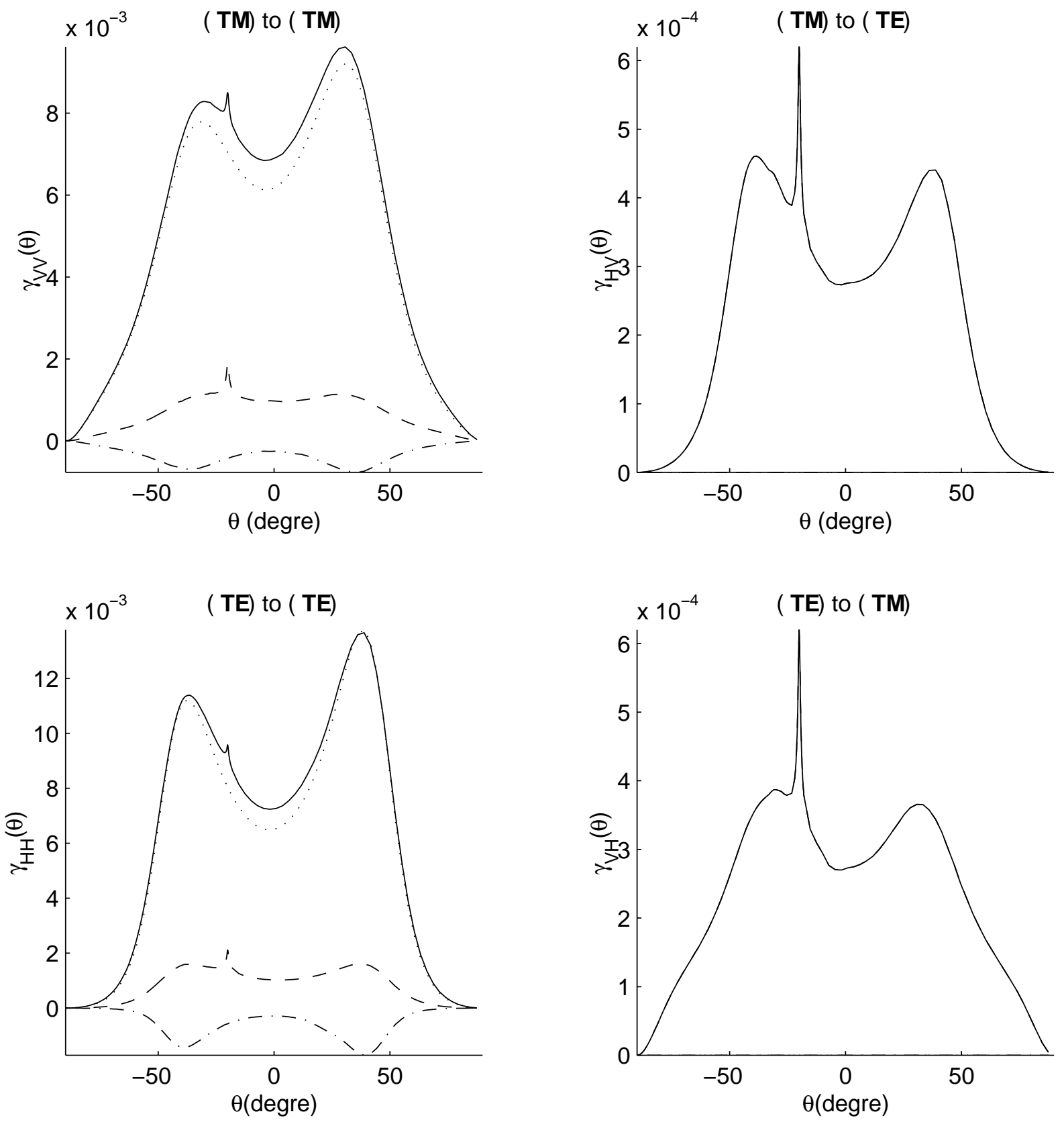

FIG. 6: Same parameters as in Fig. 田 but with $\theta_{0}=-20^{\circ}$ 

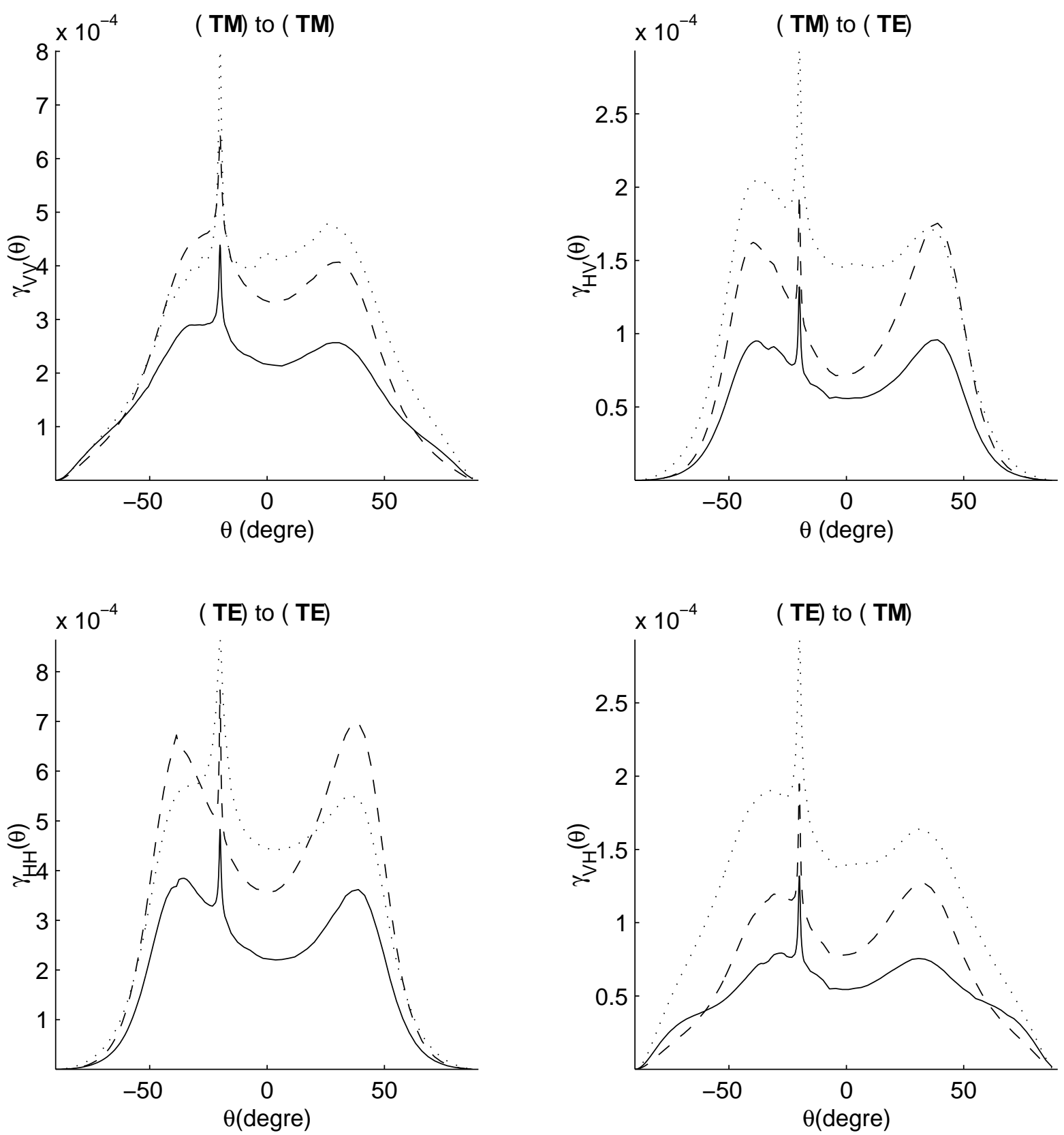

FIG. 7: Second order contributions to Fig. 6 